\title{
Optimal fluctuations and the control of chaos
}

\author{
D. G. Luchinsky ${ }^{1}$, I. A. Khovanov ${ }^{2}$, S. Berri ${ }^{1,3}$, R. Mannella ${ }^{3,1}$ \\ and P. V. E. McClintock ${ }^{1}$ \\ January 13, 2004
}

\author{
${ }^{1}$ Department of Physics, Lancaster University, Lancaster, LA1 4YB, UK. \\ ${ }^{2}$ Department of Physics, Saratov State University, Astrahanskaya 83, 410026, Saratov, \\ Russia. \\ ${ }^{3}$ Dipartimento di Fisica, Università di Pisa and Istituto Nazionale Fisica della Materia \\ UdR Pisa, Via F. Buonarroti 2, 56100 Pisa, Italy.
}

\begin{abstract}
The energy-optimal migration of a chaotic oscillator from one attractor to another coexisting attractor is investigated via an analogy between the Hamiltonian theory of fluctuations and Hamiltonian formulation of the control problem. We demonstrate both on physical grounds and rigorously that the Wentzel-Freidlin Hamiltonian arising in the analysis of fluctuations is equivalent to Pontryagin's Hamiltonian in the control problem with an additive linear unrestricted control. The deterministic optimal control function is identified with the optimal fluctuational force. Numerical and analogue experiments undertaken to verify these ideas demonstrate that, in the limit of small noise intensity, fluctuational escape from the chaotic attractor occurs via a unique (optimal) path corresponding to a unique (optimal) fluctuational force. Initial conditions on the chaotic attractor are identified. The solution of the boundary value control problem for the Pontryagin Hamiltonian is found numerically. It is shown that this solution is approximated very accurately by the optimal fluctuational force found using statistical analysis of the escape trajectories. A second series of numerical experiments on the deterministic system (i.e. in the absence of noise) show that a control function of precisely the same shape and magnitude is indeed able to instigate escape. It is demonstrated that this control function minimizes the cost functional and the corresponding energy is found to be smaller than that obtained with some earlier adaptive control algorithms.
\end{abstract}

PACS numbers: 05.45.Gg, 02.50.-r, 05.20.-y, 05.40.-a 


\section{Introduction}

The control of chaotic systems is a challenging problem that is both of intrinsic interdisciplinary interest, and also of obvious importance in relation to applications. In general the presence of noise complicates considerably any analysis of the dynamics. But we describe in what follows how a statistical analysis of fluctuations can be used to solve a problem in the nonlinear energy-optimal control of chaos. We consider the energy-optimal entraining of a nonlinear oscillator from a chaotic attractor to another coexisting attractor. Our approach is based on an analogy between the variational formulations of the deterministic control problem and the problem of fluctuations, based on the concept of the optimal fluctuational path. One of the key points is the identification of the optimal control function with the optimal fluctuational force. The solution of the energy-optimal control problem can thus be found to an excellent approximation by building the prehistory probability distribution [Dykman et al., 1992] of fluctuational escape trajectories. We compare the performance of the control function found in this way with some earlier adaptive control algorithms.

Methods already available for the control of chaos include a variety of minimal forms of interaction which are restricted by the linear approximations adopted [Shinbrot et al., 1990; Boccaletti et al., 2000; Shinbrot, 1995; Shinbrot et al., 1993; Hubinger et al., 1994; Barreto et al., 1995], and methods of strong nonlinear control [Jackson, 1997; Raj \& Rajasekar, 1997] which necessarily require a large modification of the system's dynamics, at least for limited periods. Although the latter methods have been widely used for directing the motion from a chaotic attractor (CA) to another coexisting attractor, the question of how to do so in an energy optimal way has remained an unsolved problem. Its solution must amount to an important extension of the range of model-exploration objectives (c.f. [Jackson, 1997] and [Hubinger et al., 1994]) achievable through minimal control techniques.

Consider a system of the form

$$
\dot{x}=f(x, u, t),
$$

with the state variable $x \in R^{n}$, and an admissible control function $u \in R^{m}$ in the control set $U$. We assume that it is desired to transfer the system from a given state $X_{0}=x\left(t_{0}\right)$ to a terminal state $X_{1}=x\left(t_{1}\right)$ in such a way that the ("cost") functional

$$
J=\min \int_{t_{0}}^{t_{1}} f_{0}(x, u, t) d t
$$

is minimized, with $t_{1}$ unspecified.

Let $(u(t), x(t))$ be a solution of this problem. Then there exist continuous, piece-wise differentiable functions $y_{0}(t), \ldots, y_{n}(t)$ that are not simultaneously zero and which, together with the functions $x_{i}(t)$, satisfy the differential equations (see e.g. [Hocking, 1997]) 
where the Hamiltonian is

$$
\begin{gathered}
\dot{x}_{i}=\frac{\partial H}{\partial y_{i}} \\
\dot{y}_{i}=-\frac{\partial H}{\partial x_{i}}
\end{gathered}
$$

$$
\begin{gathered}
H\left(x_{1}, \ldots, x_{n} ; y_{0}, \ldots, y_{n} ; u(t), t\right) \\
=\sum_{i=0}^{n} y_{i} f_{i}\left(x_{1}, \ldots, x_{n} ; u(t), t\right) .
\end{gathered}
$$

The optimal control function $\mathbf{u}(t)$ maximizes $H$ at each instant. $H$ is a continuous function of time and one has $H\left(t_{1}\right)=0$. If the functions $f_{i}, i=0, \ldots, n$ do not depend on time explicitly, then $H$ is constant and equal to zero.

It can be seen that finding the force that will direct the system from a chaotic attractor to another coexisting attractor with minimum expenditure of energy requires the solution of a boundary value problem for the Hamiltonian dynamics (3),(4). The difficulty in solving it stems from the complexity of the system's dynamics near a CA and relates, in particular, to delicate problems connected to the uniqueness of the solution, its behaviour near a $\mathrm{CA}$, and the boundary conditions at a CA.

In this paper we show how the energy-optimal control of chaos can be effected via a statistical analysis of the fluctuational trajectories of a chaotic system in the presence of small random perturbations. This approach is based on the analogy between the variational formulations of both problems discussed by Smelyanskiy \& Dykman [1997]: the problem of the energy-optimal control of large fluctuations, and that of the stability of an attractor weakly perturbed by random fluctuations. One of the key points of the approach is an identification of the optimal control function with the optimal fluctuational force. Brief accounts of the preliminary results [Luchinsky \& Khovanov, 1999; Khovanov et al, 2000a,b] of this approach have already been published.

We emphasize that the question of the stability of a CA under small random perturbations is itself an important unsolved problem in fluctuation theory [Kautz, 1987; Graham et al., 1991; Grassberger, 1989; Beale, 1989], and the difficulties in solving it are similar to those mentioned above. Thus it is quite unclear at first sight how an analogy between two unsolved problems can be of any help. Recently, however, a new method for the statistical analysis of fluctuational trajectories was introduced [Dykman et al., 1992, 1996; Luchinsky, 1997; Luchinsky \& McClintock, 1997; Luchinsky et al., 1998], allowing direct experimental insight into the deterministic dynamics underlying the seemingly random fluctuational motion in the limit of small noise intensity. Using this technique one can investigate experimentally the structure of the invariant manifolds in the phase space of an auxiliary Hamiltonian system arising in the analysis of both the problem of fluctuations and the problem of energy-optimal control. It turns out, in particular, that we can verify experimentally the existence of a unique solution, identify the boundary condition on the 
$\mathrm{CA}$, and find an accurate approximation for the optimal control function. The approach described below can readily be generalized to encompass systems where the boundaries of the CA's basin of attraction are fractal, and where the ergodic properties on the attractor are different.

The paper is organized as follows. In Sec. 2 we describe the particular model - a periodically driven nonlinear oscillator - formulate the control problem and write down the corresponding Pontryagin Hamiltonian. In Sec. 3 the basic ideas needed from Hamiltonian fluctuation theory are introduced and it is shown for a given model that the Hamiltonian systems arising in the analysis of both the problem of fluctuational escape and the problem of the energy-optimal control are equivalent. Sec. 5 describes and reports the results of numerical experiments to investigate fluctuational escape from the chaotic attractor showing that, in the limit of very weak noise intensity, there is a unique (optimal) escape path. Measurement of the corresponding optimal fluctuational force yields the the required optimal control function. The numerical analysis of the boundary value problem for Pontryagin's Hamiltonian is discussed in Sec. 6. It is shown that the optimal fluctuational force found from the statistical analysis of the escape trajectories provides a very accurate approximation to the solution of the boundary value problem. A second series of numerical experiments, described in Sec. 7, verifies that application of the optimal force (derived from the fluctuation experiments) brings about the desired escape from the chaotic attractor in the absence of noise and, furthermore, that the total energy used in the control process is then a minimum. The results are discussed and summarised, and conclusions are drawn, in Sec. 8.

\section{Model}

We will first formulate the energy-optimal control problem. Then we summarize briefly some relevant properties of the deterministic dynamics of the oscillator in question.

\subsection{The energy-optimal control problem}

Consider the motion of a periodically driven nonlinear oscillator

$$
\begin{aligned}
\dot{q}_{1} & =K_{1}(\mathbf{q}(t))=q_{2}, \\
\dot{q}_{2} & =K_{2}(\mathbf{q}(t))+u(t) \\
& =-2 \Gamma q_{2}-\omega_{0}^{2} q_{1}-\beta q_{1}^{2}-\gamma q_{1}^{3}+h \sin (\omega t)+u(t) .
\end{aligned}
$$

Here $u(t)$ is the control function. This particular model is of interest in number of contexts, and theoretical analysis is possible over a wide range of parameter values [Soskin et al., 
1997]. It is a system in which chaos can be observed for relatively small values of the driving force amplitude $h \approx 0.1$.

We consider the following energy-optimal control problem: How can the system (5) with unconstrained control function $u(t)$ be steered from a CA to a coexisting stable limit cycle (SC) such that the "cost" functional

$$
J_{c}=\inf _{u \in U} \frac{1}{2} \int_{t_{0}}^{t_{1}} u^{2}(t) d t
$$

is minimized? Here $t_{1}$ is unspecified and the control set $U$ consists of functions (control signals) able to move the system from a CA to the SC.

The Pontryagin Hamiltonian and corresponding equations of motion (3),(4) take the form

$$
\begin{aligned}
& \dot{q}_{i}=\frac{\partial H_{c}}{\partial p_{i}}, \quad \dot{p}_{i}=-\frac{\partial H_{c}}{\partial q_{i}}, \quad i=\{1,2\}, \\
& H_{c}=1 / 2 p_{2}^{2}+p_{1} K_{1}+p_{2} K_{2} .
\end{aligned}
$$

It is assumed that the optimal control function $u(t)$ at each instant takes those values $u(t)=p_{2}$ that maximize $H_{c}$ over $U$.

We note that for $p_{1}=p_{2}=0$ the dynamics of (7) are reduced to the deterministic dynamics of the original system (5) in the absence of any control $(u(t)=0)$. So we begin our analysis by considering some relevant properties of the deterministic dynamics of the periodically driven nonlinear oscillator (5).

\subsection{Deterministic dynamics of a nonlinear oscillator}

The parameters of the chosen system (5) were selected such that: the potential is monostable, $\left(\beta^{2}<4 \gamma \omega_{0}^{2}\right)$; the dependence of the energy of oscillations on their frequency is nonmonotonic, $\left(\frac{\beta^{2}}{\gamma \omega_{0}^{2}}>\frac{9}{10}\right)$; and the motion is underdamped, $\Gamma \ll \omega \approx 2 \omega_{0}$.

A simplified parameter space diagram obtained from the numerical simulations is shown in the Fig. 1(a). The blue dots bound the region in which both the linear and nonlinear responses of period 1 can coexist. The upper line marks the boundary of the linear response and the lower line marks that for the nonlinear responses. The boundaries of the hysteresis of the period 1 resonance are shown by yellow crosses. The region in which linear response coexists with one or two nonlinear responses of period 2 is bounded by open green circles. This region is similar to one bounded by the yellow crosses. The region of coexistence of the two resonances of period 2 is bounded by the red triangles. Chaotic states are indicated by the small black dots. The numerical investigations described in this paper where carried out for the parameter values indicated by the bold plus sign $\left(\Gamma=0.025, \omega_{0}=0.597, \beta=1, \gamma=1, \omega_{f}=0.95, h=0.13\right)$. 
A bifurcation diagram is shown in Fig. 1(b) for one set of parameters. The chaotic state appears as the result of period-doubling bifurcations, and thus corresponds to a non-hyperbolic attractor. Its boundary of attraction $\partial \Omega$ is nonfractal and is formed by the stable invariant manifold of the saddle cycle of period 1 (S1).

For a given damping $(\Gamma=0.025)$ the amplitude and the frequency of the driving force were chosen so that the chaotic attractor coexists with the stable limit cycle (SC): $h=0.13, \omega_{f}=0.95$ (see Fig. 1(a)).

The basins of attraction of the coexisting strange attractor CA and the SC are shown in Fig. 2 for a Poincaré cross-section $\omega_{f} t=0.6 \pi(\bmod 2 \pi)$ in the absence of noise. The value of the maximal Lyapunov exponent for the CA is 0.0449 .

The presence of the control function effectively doubles the dimension of the phase space (compare Eqs. (5) and (7)) and changes its geometry near the non-hyperbolic attractor. In the extended phase space the attractor is connected to the basin of attraction of the stable limit cycle via an unstable invariant manifold. It is precisely the complexity of the structure of phase space for the auxiliary Hamiltonian system (7) near the nonhyperbolic attractor that makes it difficult to solve the energy-optimal control problem.

However, as already indicated, we can apply the new method for experimental analysis of the Hamiltonian flow [Dykman et al., 1992; Luchinsky, 1997; Luchinsky \& McClintock, 1997; Luchinsky et al., 1998], exploiting the analogy between the Wentzel-Freidlin and Pontryagin Hamiltonians. To see how this can be done, we consider the fluctuational dynamics of our nonlinear oscillator (5).

\section{Hamiltonian dynamics of large fluctuations}

As pointed out above, there is an important analogy between Hamiltonians arising in the theory of fluctuations and in control theory. It arises from the small-noise asymptotic analysis of the fluctuational dynamics. We note that, for a particle interacting with a heat bath under quite general conditions, dissipative equations of motion can be related to the microscopic Hamiltonian of the system as a whole, including the heat bath and the interaction between the bath and the system. Proceeding on this basis, one arrives at the stochastic differential (or integro-differential, see e.g. [Zwanzig, 1973; Dykman \& Krivoglaz, 1984]) equations of motion

$$
\begin{aligned}
& \dot{q}_{1}=q_{2}, \\
& \dot{q}_{2}=-2 \Gamma q_{2}-\omega_{0}^{2} q_{1}-\beta q_{1}^{2}-\gamma q_{1}^{3}+h \sin (\omega t)+\xi(t), \\
& \langle\xi(t)\rangle=0,\langle\xi(t) \xi(0)\rangle=D \delta(t)=4 \Gamma k T \delta(t) .
\end{aligned}
$$

In thermal equilibrium the energy losses due to dissipation are compensated by the transfer of energy back to the system due to fluctuations, as expressed in the fluctuation-dissipation relations [Landau \& Lifshitz, 1980] which take the form $D=4 \Gamma k T$ for the system (8). 
This energy balance underlies the fact that in the zero-noise-intensity limit, a consistent theoretical development [Zwanzig, 1973; Dykman \& Krivoglaz, 1984] from the microscopic to the macroscopic equations of motion leads to descriptions of the system's deterministic (dissipative) and fluctuational dynamics within the framework of the same Hamiltonian formalism [Freidlin \& Wentzel, 1984].

If the intensity of fluctuations is small, the system spends most of the time fluctuating about the stable state, only occasionally fluctuating far away from it. In particular, noise can force the system to escape from the basin of attraction of a given stable state. These are rare events but, when they occur, they do so in an almost deterministic way: e.g. escape from a domain of attraction typically follows a unique optimal trajectory. It corresponds to an optimal (most probable) realization of the random force. The probability of any given realization of the random force for a white Gaussian noise $\xi(t)$ of strength $D$ is given by the probability density functional $P[\xi(t)]$ in the form [Feynman \& Hibbs, 1965]

$$
P[\xi(t)] \propto \exp \left(-\frac{1}{2 D} \int_{t_{0}}^{t_{1}} \xi^{2}(t) d t\right)
$$

This equation shows that in the $D \rightarrow 0$ limit the most probable (optimal) realization of the random force is given by the condition

$$
J_{n}=\inf _{\substack{\text { in } \\ q_{1}\left(t_{0}\right)=q_{i}, q_{1}\left(t_{1}\right)=q_{f}}}-\frac{1}{2} \int_{t_{0}}^{t_{1}} \xi^{2}(t) d t
$$

where the minimum is taken over all realizations $\xi(t)$ moving the system from an initial state $q_{i}$ to the final state $q_{f}$. The similarity between (6) and (10) is apparent and intuitively clear because, in thermal equilibrium $\left(D=4 \Gamma k_{B} T\right)$, the probability of fluctuations is determined by the minimum work from the external source needed to produce the given change in the thermodynamic quantities $P \propto \exp \left(-R_{\min } / k_{B} T\right)$ [Landau \& Lifshitz, 1980].

Using (8) one can transform [Feynman \& Hibbs, 1965] the "cost" functional (10) to an action integral in the form

$$
S\left(q_{1}, t\right)=\int_{t_{0}}^{t_{1}} d t L\left(q_{1}, \dot{q}_{1}, \ddot{q}_{1}, t\right)
$$

calculated along the deterministic trajectories minimizing (11), where the Lagrangian $L$ is given by

$$
L\left(q_{1}, \dot{q}_{1}, \ddot{q}_{1}, t\right)=\frac{1}{2}\left[\ddot{q}_{1}+2 \Gamma \dot{q}_{1}+\omega_{0}^{2} q_{1}+\beta q_{1}^{2}+\gamma q_{1}^{3}+h \sin (\omega t)\right]^{2} .
$$

Accordingly the escape probability takes the form $P \propto \exp (-S / D)$ and it can be seen [Onsager \& Machlup, 1953; Freidlin \& Wentzel, 1984] that in the limit of small noise intensity the large rare deviations of the system from the stable state follow the deterministic trajectories of an auxiliary classical mechanical system (12). The corresponding 
so-called Wentzel-Freidlin Hamiltonian $H_{n}$ can readily be obtained using the following relations

$$
H=\sum_{i} p_{i} \dot{q}_{i}-L, \quad p_{i}=\sum_{j=i}^{n}(-1)^{j-i} \frac{d^{j-i}}{d t^{j-i}}\left(\frac{\partial L}{\partial x^{(j)}}\right)
$$

where $n$ is the dimension of the system. For the system (12) $H_{n}$ takes a form (see e.g. [Einchcomb \& McKane, 1995])

$$
\begin{aligned}
& H_{n}=\frac{1}{2} p_{2}^{2}+p_{1} q_{2}+\left(-2 \Gamma q_{2}-\omega_{0}^{2} q_{1}-\beta q_{1}^{2}-\gamma q_{1}^{3}+h \sin (\omega t)\right) p_{2} \\
& p_{i}=\frac{\partial S}{\partial q_{i}}, \quad \mathrm{i}=1,2 .
\end{aligned}
$$

It follows from (11) and (13) that the function $S\left(q_{1}\right)$ satisfies the Hamilton-Jacobi equation in the form

$$
S_{t}+H\left(\mathbf{q}, \frac{\partial S}{\partial \mathbf{q}}, t\right)=0
$$

where the Hamiltonian $H$ is given by (14). $S\left(q_{1}\right)$ is non-decreasing along the optimal fluctuational trajectories. Although $S\left(q_{1}\right)$ is in general not differentiable, it can be proven [Freidlin \& Wentzel, 1984] that it satisfies a local Lipshitz condition, which implies differentiability almost everywhere and justifies the use of this function as a generalized Lyapunov function for the deterministic system (14) [Jauslin, 1987].

The trajectories of (14) minimize the action $S\left(q_{1}, t\right)$ in (11). In general there will be many such trajectories that begin at $q_{i}, t_{i}$ and terminate at $q_{f}, t_{f}$. As a consequence, the quantity $S\left(q_{1}, t\right)$ is in general multivalued. Trajectories providing a global minimum for the action are called optimal fluctuational paths (see also e.g. [Maier \& Stein, 1993; Dykman et al., 1994a,b; Maier \& Stein, 1996; Dykman et al., 1996; Maier \& Stein, 1997; Smelyanskiy et al., 1997] and discussion therein). The action calculated along the optimal fluctuational paths for the system (14) is sometimes called a generalized nonequilibrium potential (see e.g. [Graham \& Tel, 1984; Jauslin, 1987]). For the system (14) in thermal equilibrium $(h=0)$ the fluctuational trajectories become time-reversed images of the relaxational trajectories (see e.g. [Onsager \& Machlup, 1953]) and as a consequence the nonequilibrium potential is reduced to the equilibrium one.

It is clear that the Hamiltonians (7) and (14) coincide. The analogy between two problems opens the possibility of direct experimental insight into the geometry of the phase space of system (4) using a statistical analysis of the fluctuational trajectories in this system when a control function $u(t)$ is substituted for the random function $\xi(t)$. In particular, the optimal control function $\bar{u}(t)$ can be identified with the optimal fluctuational force which drives the system from the chaotic attractor to the stable limit cycle [Smelyanskiy \& Dykman, 1997]. We note that both $\bar{u}(t)$ and the optimal force are related to $p_{2}$ in $(7)$ and (14). 
We have therefore suggested that the optimal control function $\bar{u}(t)$ can be found experimentally by measurement of the optimal fluctuational force [Luchinsky, 1997; Luchinsky et al., 1998].

We emphasise that the analysis in this section and in Sec. II(A) draws an analogy between two seemingly separate problems: the deterministic energy-optimal control problem and the problem of stability in the presence of small random perturbations. However, similar conclusions can be drawn using a more general formulation of the stochastic optimal control problem (see e.g. [Smelyanskiy \& Dykman, 1997]) as discussed briefly in Sec. 7.

It is of central imoortance that: (i) the Hamiltonian dynamics of large fluctuations can, at least in some cases [Zwanzig, 1973; Dykman \& Krivoglaz, 1984], be related directly to the microscopic classical equations of motion for a system interacting with a heat bath; (ii) the corresponding formalism includes both the dynamics of relaxation toward the stable state and the dynamics of a fluctuation-induced large deviation from this state on equal grounds; (iii) the motion described by the corresponding Hamiltonian equations is experimentally observable [Luchinsky, 1997; Luchinsky \& McClintock, 1997; Luchinsky et al., 1998]; experimental investigations of the Hamiltonian dynamics of large fluctuations can be used to solve the energy-optimal control problem.

\section{Statistical analysis of fluctuational trajectories}

The statistical analysis of the fluctuational trajectories is based on measurements of the socalled prehistory probability distribution introduced by Dykman et al. [1992], $p_{h}\left(\mathbf{q}, t ; \mathbf{q}_{f}, t_{f}\right)$. Here $p_{h}$ gives the probability for the system to pass through the point $\mathbf{q}$ at the instant $t$ under the condition that it departs from the CA at $t=-\infty$ and arrives at the final point $\mathbf{q}_{\mathbf{f}}$ at $t=t_{f}$. Note that it differs from the ordinary conditional probability distribution and that it sets optimal paths into a physical context: their physical significance follows from the fact that $p_{h}\left(\mathbf{q}, t ; \mathbf{q}_{f}, t_{f}\right)$ at any given moment of time $t$ should have a sharp maximum in $\mathbf{q}$ lying on the optimal path, $\mathbf{q}=\mathbf{q}_{\mathrm{opt}}\left(t \mid t_{f}, \mathbf{q}_{f}\right)$; and the optimal path is in fact just the $D \rightarrow 0$ limit of the ridges of the prehistory distribution. By investigating the prehistory probability distribution experimentally, one can establish the region of phase space within which optimal paths are well defined, i.e. where the tube of fluctuational paths around an optimal path is narrow. The prehistory distribution thus provides information both about the optimal path and about the probability that it will be followed. In experiments the technique involves following the dynamics of the system continuously and constructing the distribution of all realizations of fluctuational trajectories that transfer the system from a state of equilibrium to the prescribed remote state. The advantages of this approach have been demonstrated previously [Luchinsky, 1997; Luchinsky \& McClintock, 1997; Luchinsky et al., 1998] in the course of investigations of the fundamental symmetry 
properties of optimal trajectories and singular characteristics of their distribution.

To find the optimal control function $\bar{u}(t)$ we have performed digital simulations of (8) using the Heun algorithm, with particular attention being paid to the random number generator (see [Mannella, 1997; Marsaglia \& Tsang, 1984]), because simulation times necessarily grow exponentially as $D \rightarrow 0$. We have also carried out a complementary analog electronic modeling [Luchinsky et al., 1998] of (8). We drive the model with zero-mean quasi-white Gaussian noises from a noise generator, digitize the response $q_{1}(t), q_{2}(t), \xi(t)$, and analyze it with a digital data processor. In both the analog and digital simulations, trajectories moving the system from the chaotic attractor to the stable limit cycle were collected, and the corresponding distributions of escape trajectories were built and analyzed. Qualitatively similar results were obtained but, because precision is of particular importance here, most of the data reported below are those from the digital simulations.

For the technique to be applicable, a solution of (7) moving the system from the CA to $\partial \Omega$ must of course exist, and one has to be able to identify the boundary conditions for this solution on the CA.

\subsection{Escape scenario}

In the presence of weak noise there is a finite probability of noise-induced transitions between the chaotic attractor and the coexisting stable limit cycle. In Fig. 2 the small filled circles show the intersections of one of the actual escape trajectories with the given Poincaré section. We now discuss the escape scenario that is intuitively to be expected on the basis of the Hamiltonian formalism.

We consider first escape of the system from the basin of attraction of a stable limit cycle that is bounded by a saddle cycle. In general, escape occurs along a single optimal trajectory $q_{\text {opt }}(t)$ connecting the two limit cycles. The trajectory $q_{\text {opt }}(t)$ is determined by minimizing $S$ in (11) over the set of all classical deterministic trajectories determined by the Hamiltonian $H$ (14) that start on the stable limit cycle as $t \rightarrow-\infty$ and terminate on the unstable limit cycle as $t \rightarrow \infty$. That is, $q_{\text {opt }}(t)$ is a heteroclinic trajectory of the system (14) with minimum action, where the minimum is to be understood in the sense indicated above. We note that the existence of optimal escape trajectories and the validity of the Hamiltonian formalism have been confirmed experimentally for a number of nonchaotic systems (see [Luchinsky, 1997; Luchinsky \& McClintock, 1997; Luchinsky et al., 1996, $1998,1999]$ and the references cited therein). If the noise is weak, then the probability $P \approx \exp (-S / D)$ of escape along the optimal trajectory is exponentially small, but it is exponentially greater than the escape probability along any other trajectory, including along other heteroclinic trajectories of the system (14).

We next consider escape from a chaotic attractor. Because the basin of attraction of the $\mathrm{CA}$ is bounded by an unstable limit cycle S1, the situation near S1 remains qualitatively the same and the escape trajectory remains unique in this region. However, the 
situation is different near the chaotic attractor itself. In this region it is virtually impossible to analyze the Hamiltonian dynamics of the auxiliary system (14), and no predictions have been made about the character of the distribution of optimal trajectories near the CA. The simplest scenario is that an optimal fluctuational trajectory approaching (in reversed time) the boundary of a chaotic attractor is smeared into a "cometary tail" and is lost, merging with the boundary of the attractor.

However, a statistical analysis of measured fluctuation-induced escape trajectories gives a different and more detailed picture of what actually occurs during noise-induced escape from a chaotic attractor. Several thousand escape trajectories of the system (8) from the basin of attraction of its CA have been measured for a variety of different operating regimes. The typical situation as measured in the analog and digital simulations is displayed in Fig. 3(a) for system parameters close to the point $\mathrm{P}$ in Fig. 1 and a noise intensity $D \approx 0.0005$. The figure shows 65 measured fluctuational escape trajectories, each of which has been shifted in time so that the regions corresponding to the transition from chaotic to regular motion coincide with each other.

It is evident that all the trajectories end up close together, passing through the immediate neighborhood of some optimal trajectory within a tube of radius $\propto \sqrt{D}$; at earlier time, however, they separate into distinct groups. It is therefore possible to determine the optimal escape paths by simple ensemble-averaging, separately for each group of trajectories. Three optimal paths determined from the digital simulations are shown in Fig. 3(b). Analysis of the optimal trajectories shows that fluctuation-induced escape from the boundary of the basin of attraction of the CA is characterised by the following features-

(i) The system may escape from the CA along any one of several nearly optimal trajectories.

(ii) These merge into a single trajectory approaching the saddle cycle S3 located near the CA.

(iii) The final escape occurs along a single optimal escape trajectory connecting S3 and the saddle cycle S1 located at the boundary of the basin of attraction of the CA.

The number of optimal trajectories of the transition $\mathrm{CA} \rightarrow \mathrm{S} 3$ depends on the choice of the working point. Usually, between one and three escape trajectories were observed experimentally. The escape probabilities along them differ (see, caption to Fig. 3(b). If the noise intensity is reduced further, one of the escape paths becomes exponentially more probable than all the others. In what follows we concentrate on the properties of this most probable escape path. 


\subsection{Boundary conditions}

To find the boundary conditions at the CA we analyze the prehistory probability distribution $p_{h}\left(\mathbf{q}, t ; \mathbf{q}_{f}, t_{f}\right)$ of the escape trajectories. The measured distribution is shown in Fig. 4. By inspection of how the ridge of the most probable escape path merges with the $\mathrm{CA}$, it can be inferred that most of the escape trajectories pass close to the saddle cycle of period 5 embedded in the CA.

This inference can be elaborated further using the fact that all the measured escape trajectories merge the saddle cycle of period 3 and that this cycle is situated near the attractor. It is therefore possible to perform a statistical analysis of the trajectories arriving in a small tube around S3, with the temperature reduced by a few orders of magnitude, to $T=1.5 \times 10^{-6}$. The corresponding trajectories are shown in Fig. 4, from which it can be seen that: (i) there is indeed only one optimal path connecting the CA to the saddle cycle S3 at lower temperatures; and (ii) the system escapes from the chaotic attractor via the saddle cycle S5.

Finally, we use the generalized monodromy technique of Simonovic [1999] to verify that, given initial conditions on the actual escape trajectory passing close to S5, this method converges to S5. Simultaneously with convergence to S5 this method produces the monodromy matrix of the cycle. The corresponding multipliers are $m_{1}=4.5643$, $m_{2}=0.0420$.

\subsection{Approximate solution of the energy-optimal control prob- lem}

The above statistical analysis of escape trajectories reveals that the energetically most favorable way of moving the system from the CA to the stable limit cycle starts at the saddle cycle S5 embedded in the CA, passes through the saddle cycle S3, and finishes at the saddle cycle S1 marking the boundary of the basin of attraction of the CA. Further motion of the system towards the stable limit cycle does not require external action.

To find an approximation to the optimal control function we collect all successful realisations $\left(q_{1}^{\text {esc }}(t), q_{2}^{\text {esc }}(t), \xi^{\text {esc }}(t)\right)$ corresponding to passage from S5 to $\partial \Omega$. An approximate solution $\tilde{u}(t)$ is then found as an ensemble average over the corresponding realisations of the random force $\left\langle\xi^{\operatorname{esc}}(t)\right\rangle$ (the exact solution is $\bar{u}(t)=\lim _{D \rightarrow 0} \tilde{u}(t)$ ). The results of this procedure are shown in the upper part of Fig. 6 where, to minimise the irrelevant high-frequency component still remaining after the averaging procedure, we have applied a zero-phase low-pass filter with a high frequency cut of $\omega_{\text {cut }}=1.9$. It can be seen from the figure that the optimal force switches on at the moment when the system leaves S5 along its unstable manifold. The optimal force returns to zero again when the system reaches the saddle cycle $\mathrm{S} 1$.

Thus we conclude that the solution $\tilde{u}(t)$ and the corresponding boundary conditions 
can indeed be found by use of our new experimental method. Moreover the problem of escape from the $\mathrm{CA}$ of a periodically driven nonlinear oscillator evidently reduces to the analysis of transitions between the three saddle cycles $\mathrm{S} 5 \rightarrow \mathrm{S} 3 \rightarrow \mathrm{S} 1$. We note that the latter result is in qualitative agreement with the well known statement that unstable cycles provide detailed invariant characterizations for dynamical systems of low intrinsic dimension (see e.g. [Auerbach et al., 1987; Grebogi et al., 1988; Schmelcher \& Diakonos, 1997]). This result opens up the possibility of numerical solution of the corresponding boundary value problem for the energy-optimal control formulated in Sec. 2.

\subsection{Finite noise intensity}

An essential feature of the experimental technique outlined above is the presence of noise of finite intensity $D$. The later introduces some changes to both the energy-optimal control problem and the dynamics of the system on the CA. Therefore, if we are interested in finding an approximate solution of the deterministic control problem, there are at least two key questions we need to address: (i) to what extent is the observed form of the most probable escape path independent of the initial conditions on the strange attractor; (ii) how accurate is the approximate solution?

\subsubsection{Natural measure on the attractor}

To demonstrate that the measured fluctuational force is an approximation to the true stationary optimal control function one has to show that the shape of the optimal force does not depend on the initial conditions on the attractor. In particular, this means that the characteristic time required for the system to approach S5 from an arbitrary point inside the attractor basin $\left(t_{r}\right)$ should be much shorter then the average time of noise-induced escape from the basin of attraction $\left(t_{e}\right)$.

The time required for the system to approach the saddle cycle S5 is typically [Grebogi et al., 1988; Dhamala \& Lai, 1999]

$$
t_{r}=\frac{1}{p_{i}(l)} \propto l^{-D_{p}}
$$

where $p_{i}(l)$ is a natural measure contained in a small square of unit size $l$ centered on S5 in a Poincaré cross-section and $D_{p}$ is the pointwise dimension of S5.

To analyze the behaviour of the natural measure as a function of noise intensity, the portion of the Poincaré cross-section containing the attractor was partitioned into squares $\left\{S_{i}\right\}$ of unit size $l$. The $p_{i}$ were measured as the fraction of time the trajectory originating at an arbitrary initial condition $\bar{x}$ spends in the vicinity of each $S_{i}$ : $p_{i}=$ $\lim _{N \rightarrow \infty} N_{i} / N$, where $N$ is the number of periods of the driving force corresponding to the total time of observation and $N_{i}$ is the number of periods the trajectory is found in $S_{i}$. The measurements were performed for the limit cycle S5 and for two other saddle 
cycles embedded into the chaotic attractor of period 2 (S2) and period 8 (S8). The later two cycles are situated in the region where the stationary probability distribution on the attractor in the presence of fluctuations has maximum.

The pointwise dimensions obtained from a linear fit of the $\log -\log$ dependence of $p_{i}$ on $l$ are 1.5327, 1.2148 and 1.3396 for S2, S5 and S8 respectively. A conjecture by Grebogi et al. [1988] relates $D_{p}$ to the magnitudes of the unstable and stable eigenvalues $\lambda_{1}>1$ and $\lambda_{2}<1$ of the Jacobian matrix of the vector field linearised about corresponding limit cycles $D_{p}=1-\log \lambda_{1} / \log \lambda_{2}$. For future reference we give here the values of $D_{p}$ calculated according the formula above: 1.5344, 1.4800, 1.5132 for the cycles S2, S5 and S8 respectively. We note that there is a some correlation between the calculated and measured eigenvalues, although further research and better statistics will be needed to analyze this correlation.

The average time for the system to approach S5 is, according to (16), of the order of $t_{r} \approx 10^{4}$ for a box size of $l=0.002$. For non-zero temperatures the distribution becomes more uniform, and the pointwise dimension increases towards the limiting value $D_{p}=2$. Correspondingly, the relaxation time increases to $t_{r} \approx 10^{5}$. These estimates are consistent with direct measurements of the dependence of $p_{i}$ on $N$ for different values of $T$.

The average escape time on the other hand is of the order of $t_{e} \approx 10^{8}$ for a typical value of the temperatures used in our simulations, $T=0.001$.

Thus we conclude that the optimal escape path found from a statistical analysis of the escape trajectories is independent of the initial conditions on the attractor and provides an approximation to the global minimum of the corresponding deterministic control problem. However, the problem of behaviour of the natural measure of a non-hyperbolic attractor in the presence of noise is an open problem of fundamental importance (see e.g. [Anishchenko et al., 2000] and references therein). And we emphasize the preliminary character of the empirical results reported in this section.

\subsubsection{Stochastic control problem}

An analysis of the accuracy and convergence of the approximate solution to the exact one requires a more general formulation of the stochastic control problem which goes beyond the scope of the present paper, see e.g. [Fleming, 1978; Whittle, 1996; Smelyanskiy \& Dykman, 1997] and references therein. However, as concerns the control of switching probabilities the main conclusion of earlier work [Smelyanskiy \& Dykman, 1997] was that the in the limit of small noise intensity the control function converges to the optimal fluctuational force. Here we provide some additional semi-quantitative arguments to illustrate this idea and to gain further insight into the analogy between two problems.

Let us consider a stochastic version of the problem formulated in the Introduction (following e.g. [Dreyfus, 1965]) of the form

$$
\dot{x}_{i}=f_{i}(x, u, t)+\xi_{i}(t), \quad i=1, . ., n,
$$




$$
\left\langle\xi_{i}(t)\right\rangle=0, \quad\left\langle\xi_{i}(t) \xi_{j}(0)\right\rangle=D \delta_{i j} \delta(t) .
$$

We seek that control function $u(x, t)$ which transfers the system from a given state $X_{0}=$ $x\left(t_{0}\right)$ to a terminal state $X_{1}=x\left(t_{1}\right)$ and minimizes the functional

$$
J[u]=E\left[\int_{t_{0}}^{t_{1}} f_{0}(x, u, t) d t+\Phi\left(t_{1}\right)\right] .
$$

Here $E$ denotes a mathematical expectation with respect to the joint probability function of random variables $\xi_{m}(t)$. The later are assumed for simplicity to be Gaussian independent variables of equal strength $D . \Phi\left(t_{1}\right)$ is a given terminal boundary condition for the expected value function defined by the following relation

$$
\tilde{S}(x, t)=\min _{u} \underset{\xi}{E}\left[f_{0}(x, u, t) \Delta t+o(\Delta t)+\tilde{S}(x(t+\Delta t), t+\Delta t)\right] .
$$

In (19) only $f_{0}$ is optimized with respect to the control function while $\tilde{S}(x(t+\Delta t), t+\Delta t)$ is known and optimized over $u, t$ is a given instant of time $t_{0}<t<t_{1}$, and $\Delta t$ is a small time step. Expanding $S$ on the right hand side, assuming that the third derivatives of $S$ are bounded, and collecting terms $\propto \Delta t$ yields [Dreyfus, 1965] an equation

$$
0=\min _{u}\left[f_{0}(x, u, t)+\tilde{S}_{t}+\sum_{i=1}^{n} \tilde{S}_{x_{i}} f_{i}+\frac{D}{2} \sum_{i=1}^{n} \sum_{j=1}^{n} \tilde{S}_{x_{i} x_{j}}\right]
$$

that, with boundary conditions $\tilde{S}\left(x\left(t_{1}\right), t_{1}\right)=\Phi\left(t_{1}\right)$, provides a solution for the Brownian motion control process. It can be seen from Eq. (20) that in the presence of weak noise $D$ determines the width of the distribution about the optimal path and thus defines the accuracy of the solution.

For small noise intensities $D$ the last term in the Eq. (20) can be neglected. Defining a classical action $S(x, t)$ as $y_{0} \tilde{S}(x, t)$ (where $y_{0}$ is an arbitrary negative constant) the solution of (20) is reduced to the analysis of Pontryagin's Hamiltonian system (3) and (4). We see from Eq. (19) that, for the energy-optimal control $\left(f_{0}=\frac{1}{2} u^{2}\right) S(x, t)=y_{0}, \tilde{S}(x, t)$ is a nondecreasing function along the optimal path and thus that it can be considered as a generalized Lyapunov function of the corresponding deterministic problem.

\section{$5 \quad$ Numerical solution of the boundary value problem}

It was mentioned in the introduction that finding the optimal control function requires the solution of a boundary value problem for the Hamiltonian dynamics (3),(4). But even for this relatively simple system, the solution represents a formidable task. First, the boundary conditions are in general unknown, in particular those applying at the starting time (which belong to the strange attractor). But even if the boundaries were known, the functional $R$ of Eq. (6) has so many local minima that it has proved impractical to attempt a general search for the optimal path. 
However, a statistical analysis of the fluctuational trajectories provides the a priori information needed to calculate the optimal path from Pontryagin's Hamiltonian. This approach exploits the escape scenario that had emerged from the experiments: as mentioned above, the optimal escape trajectory starts at S5 and ends at S1, passing through S3. We therefore built an initial trial function such that at short times it coincides with S5, while at large times it coincided with S1. At intermediate times, we had a mixture of S5 and S1 in the initial trial function, but no S3. Using a well known algorithm for two point boundary conditions (TWPBVP, obtained via netlib ${ }^{1}$ see [Cash, 1986, 1988; Cash \& Wright, 1990, 1991] for details), we then relaxed this trial function to find the optimal path, defined as the path which minimises the functional (6). It is striking that the relaxed optimal path that we found does go through S3, in good agreement with the optimal path obtained by ensemble averaging fluctuational trajectories and building the prehistory distribution.

The result is shown in Fig. 7(a). The blue dashed lines are examples of the three different initial trial functions. All the different trial functions relax to the same optimal function shown by the green dotted line. The red solid line shows for comparison the optimal fluctuational trajectory. We should add that we checked the robustness of this solution, changing the time range over which the solution was sought, changing the weight of S5 and S1 in the trial function and, finally, replacing S1 with the stable limit cycle: we always relaxed to the same optimal function.

From the figure, it is clear that agreement with the fluctuational optimal control function is good, albeit not perfect especially at short times. We note, however, that in this region both of the methods used - statistical analysis of the escape trajectories and numerical solution of the boundary value problem - have limited accuracy. The accuracy of the first method is limited by the finite values of $D$. The possible errors of the second method may be attributed to the boundary conditions: we fixed the starting and ending boundary conditions exactly on S5 and S1, respectively. The latter is only an approximation because the dynamical system approaches these structures, in theory, only in the limit of large times so that, after a finite time, it cannot be identically on the structure itself. Work is in progress to shift the boundary conditions away from S5, S1 to their neighborhoods at finite times.

\section{Energy-optimal migration control}

In this section we analyse the problem of migration control in our periodically driven nonlinear oscillator in the absence of fluctuations. We demonstrate that an application of the approximate solution found from the statistical analysis of the fluctuational trajectories is indeed optimal in the sense that it minimises the energy of the control function.

\footnotetext{
${ }^{1}$ Codes can be obtained from http://www.netlib.no/.
} 
The dependence of the energy on the shape of the control function, and on the boundary conditions, is investigated via digital simulations. And we compare the performance of some known adaptive control algorithms with that of the control function found in our analysis.

\subsection{The algorithm for energy-optimal control}

To verify that the force $\tilde{u}(t)$ found in the experiment does minimize the energy of the control function steering the system (5) from the CA to the S1, we set the system to arbitrary initial conditions in the basin of attraction of its CA and let it evolve deterministically until it passed through the initial part of the unstable manifold of S5. At this moment the deterministic control function was switched on. For small variations in the shape of the control function and/or initial conditions, the amplitude of the control function was set to the threshold at which switching of the system from chaotic motion to regular motion on the stable limit cycle occurred.

It was found that the system is very sensitive to variations in either the shape of the control function or the initial conditions: any deviation from the shape of $\tilde{u}(t)$, or from the initial conditions found in the experiment, leads to a substantial increase in the energy of the control function required to steer system from the CA to S1. Some experimental results are shown in Fig. 8(a) and (b). Thus it can be seen that the energy of the control function becomes approximately twice larger if the optimal force is approximated by a sin function modulated by a Gaussian, $u(t)=a_{1} \sin \left(a_{2} t\right) \exp \left(-\left(t-a_{3}\right)^{2} a_{4}\right)$, and that it becomes respectively $\sim 4$ and 20 times larger if the optimal force is approximated by rectangular pulses, or perturbed with arbitrary low-frequency perturbations.

\subsection{The OPCL control method}

For comparison, we have also performed experiments using the open-plus-closed-loop control technique [Jackson, 1997] and an adaptive control algorithm [Raj \& Rajasekar, 1997] to steer the system from the CA to S1. The equations of motion can be written in the form

$$
\begin{aligned}
& \dot{q}_{1}=q_{2}+F_{1}(q, g, t), \\
& \dot{q}_{2}=-2 \Gamma q_{2}-\omega_{0}^{2} q_{1}-\beta q_{1}^{2}-\gamma q_{1}^{3}-f \cos (\omega t) \\
& +F_{2}(q, g, t), \quad q=q_{1}, q_{2}, \quad g=g_{1}, g_{2}
\end{aligned}
$$

Here $F(q, g, t)$ is the control function

$$
F(q, g, t)=(\dot{g}-K(g))+S(t)\left(K^{\prime}(g)-A\right)(g(t)-q(t))
$$

We will be interested in the situation when the "goal dynamics" $g(t)$ is a solution of (5) with $u(t)=0$, i.e. $\dot{g}=K(g)$. Namely $g(t)$ describes the stable limit cycle of period 1 , 
$\mathrm{SC}$, coexisting with the CA. Thus the first term in (22) vanishes, and $F(q, g, t)$ takes the following explicit form

$$
F_{i}(q, g, t)=S(t) \sum_{j=1,2}\left(K_{i j}-a_{i j}\right)\left(g_{j}-q_{j}\right)
$$

Here $i=1,2$ and $K_{i j}=\partial K_{i} / \partial q_{j}$. We have considered only the case $a_{i j}=-\left|a_{i j}\right| \delta_{i j}$ and $S(t)=1-\exp (-\lambda t)$ as suggested by Raj \& Rajasekar [1997]. Parameters $|a|$ and $\lambda$ were varied so as to minimise the energy of the control function.

The energies of the control functions obtained by these methods vary from 0.14 to 0.6. Thus they are more than an order of magnitude larger then the energy of the optimal control function $\tilde{u}(t)$ found by our new technique. The dynamics of the system in the presence of OPCL control, and the shape of corresponding control functions, are shown in Fig. 9(a) and (b) respectively. The integration was stopped after the system had crossed

the saddle cycle of period 1 , at the boundary of the basin of attraction because, as mentioned above, its further evolution towards the stable limit cycle requires no external action. The energy of the control function in question (which we note has two components) is compared to the energy of our optimal control function in Fig. 8(b).

\subsection{The algorithm for adaptive control of chaos}

The migration of the nonlinear oscillator from the CA to SC with a single control function in the form of (5) was also effected through application of the adaptive chaos control algorithm proposed by Boccaletti et al. [2000].

Just as in the case of the OPCL method the control function at every time step is proportional to the difference between the goal dynamics $g(t)$ and the current state $q(t)$

$$
u\left(t_{n}\right)=K\left(t_{n}\right)\left(g\left(t_{n}\right)-q\left(t_{n}\right)\right)
$$

where the coefficient of proportionality is given by

$$
\begin{aligned}
& K\left(t_{n}\right)=K_{0} /\left(1-\tanh \left(\sigma \lambda\left(t_{n}\right)\right)\right), \\
& \lambda\left(t_{n}\right)=\ln \left(\left|\frac{g\left(t_{n}\right)-q\left(t_{n}\right)}{g\left(t_{n-1}\right)-q\left(t_{n-1}\right)}\right|\right) .
\end{aligned}
$$

The coefficients $K_{0}>0$ and $\sigma>0$ were varied in the digital simulations so as to minimize the energy of the control function. The migration dynamics and the corresponding control function are shown in Fig. 10(a) and (b), and its energy is compared to that of the optimal control in Fig. 8(b).

We note in conclusion of this section that neither the OPCL nor the adaptive control algorithm were devised to minimize the energy of the control pulse, but rather the recovery time. It is clear that these methods are insensitive to the initial conditions on the CA. The shapes of the control functions are to a large extent prescribed by the algorithms and 
are not optimized. In this sense the relatively high energy of the control functions is not a surprise. The results presented in the last two subsections illustrate compellingly the great sensitivity to the shape of the control function as well as to the initial conditions discussed above.

\section{Discussion}

We have found a novel technique for determination of the optimal control force needed to steer a nonlinear oscillator away from the basin of attraction of a non-hyperbolic strange attractor. The technique applies to the case where the cost functional, referred to as the "energy" of the control force, is quadratic in the control force.

Our approach is based on the formal correspondence that exists between the solution of the deterministic optimal control problem and the problem of escape from the attractor under the action of a zero-mean, delta-correlated, Gaussian random force that substitutes for the control force in the equations of motion. In the limit where the noise intensity goes to zero, the form of the most probable realization of the random force that effects escape of the system from the attractor approaches the optimal form of the control force in the deterministic equations of motion, i.e. the force that minimizes the total energy of the control pulse. Similarly, the activation energy for escape approaches the optimal control energy in this limit.

The interrelation between the theory of large deviations and that of optimal control was considered by Fleming [1978] and Smelyanskiy \& Dykman [1997] (see also [Whittle, 1996] and references therein). However, the experimental technique presented in the present paper is new. We use it to determine the optimal control force from an analysis of the escape trajectories using the prehistory probability distribution [Dykman et al., 1992]. If a unique solution (or a finite number of solutions of approximately equal probability) exists, the prehistory distribution peaks sharply around the most probable escape path (or around a few nearly equally probable escape paths). In this case the solution can be found by statistical averaging of the observed realizations of the random force leading to the escape events.

The technique opens up a new avenue for the study of complex nonlinear control problems where an analytical solution is impossible. Its main significance lies in the fact that it enables one to obtain fundamental information about the globally optimal control function merely by observing the system's natural behavior in the presence of noise. Once the boundary conditions for the control problem are identified, and the form of the solution has been qualitatively understood from the simulations, one can obtain the optimal control solutions for any given choice of system parameters using standard methods, as demonstrated above.

Note that in studying complex nonlinear control problems, similar to the one consid- 
ered here, conventional numerical methods require a priori knowledge of the initial and final states of the system corresponding to the optimal control solution, and thus these methods would have to be used in conjunction with the stochastic technique described in the paper. Moreover, even when the boundary conditions are identified, the determination of the optimal path is in practice a formidable task because the cost functional has many local minima and it is often impossible to attempt a search for the global minimum. In this situation an initial trial function can also be found using the stochastic technique as described.

The stochastic technique described in the paper can profitably be combined with known "minimal" forms of control and it extends substantially the variety of modelexploration objectives that can be achieved by this methods. For example, it was shown recently [Feudel \& Grebogi, 1997; Feudel et al., 1998; Kraut et al., 1999; Gadaleta \& Dangelmayr, 2001] that the task of controlling complexity in multistable systems goes far beyond the original [Shinbrot et al., 1990] control-of-chaos idea. As well as stabilising the system on one of the attractors, one needs to control switching between them. Thus far, new switching strategies have been restricted to the possibility of linearising the flow in the neighbourhood of the stable state [Feudel \& Grebogi, 1997]. The efficiency of switching can be significantly increased by the combination of the linear control algorithms with migration control methods [Raj \& Rajasekar, 1997; Jackson, 1997] or with our novel energy-optimal switching technique.

As already mentioned, the migration control algorithms were devised to minimise the recovery time, rather than the control energy. But the possibility of reducing the energy of the switching pulse by an order of magnitude, through our new technique, may have important advantages in application to the control of chaos in multistable systems, e.g. in laser systems as discussed by [Chizhevsky et al., 1997], by [Chizhevsky \& Turovets, 1994], and by [Corbalán et al., 1995].

We note also that the time required for the system to approach the unstable manifold (the saddle limit cycle S5) varies for different initial conditions on the CA and is typically $l^{-D_{p}}$, where $l$ is the linear dimension of the region and the $D_{p}$ is the pointwise dimension of the periodic point in his region [Grebogi et al., 1988]. In order to reduce this waiting time, our approach can again be combined with the technique that [Barreto et al., 1995] developed earlier for targeting the system on a given unstable periodic orbit embedded in a CA.

There are a number of important questions related to further application of this technique that require detailed investigation. A possible extension of the method to problems in higher-dimensions needs to be analysed in detail for each particular case. No general recipe can be given at this stage; but we comment that the analysis of lower dimensional cases, as here, is likely to provide a useful guide. We also comment that extension to higher dimensions will require a corresponding extension to maps of the technique for 
analysing optimal fluctuational trajectories - which can be done, at least in principle: see e.g. [Graham et al., 1991; Grassberger, 1989; Kifer, 1990b,a].

A very important problem is that of the ergodicity of the attractor. In the limit of very weak noise it may require, in general, an exponentially long time to communicate between different parts of the chaotic attractor. In this sense the deterministic control problem of switching from a strange attractor is fundamentally ill-posed at zero noise intensity. However, in the presence of finite external noise [Fleming, 1978; Smelyanskiy \& Dykman, 1997] one can define the problem of optimal control. In this case it is guaranteed that at some noise level the transit time between different points of the strange attractor is small compare to the escape time from the attractor.

Preliminary investigations [Khovanov et al, 2000b] demonstrate that the method can be extended to the analysis of escape from the Lorenz attractor. They have shown that ergodicity can be a real problem in such cases, where the optimal escape path follows the separatrix of the saddle point, which cannot be reached experimentally during a finite time in the absence of noise. In the particular case of the Lorenz attractor, the transit time has to be considered explicitly. It has recently been demonstrated, however, that the method can also reveal non-stationary optimal escape trajectories [Arrayás et al., 2000], i.e. trajectories that minimize the escape energy on a fixed time interval.

In summary, a novel technique for solution of the energy optimal control problem of steering a nonlinear oscillator from the basin of attraction of a CA has been proposed and verified experimentally. We believe that the technique can readily be extended to the treatment of more complex cases.

\section{Acknowledgements}

It is a pleasure to acknowledge valuable discussions with V.S. Anishchenko, M.I. Dykman, V.N. Smelyanskiy, and S.M. Soskin. The research has been supported in part by the Engineering and Physical Sciences Research Council (UK), INTAS, the EC, the Royal Society of London, and by Award No. REC-006 of the U.S. Civilian Research \& Development Foundation for the Independent States of the Former Soviet Union (CRDF). 


\section{References}

Anishchenko, V. S., Kopeikin, A. S., Vadivasova, T. E., Strelkova, G. I. \& Kurths, J. [2000] "Influence of noise on statistical properties of nonhyperbolic attractors", Phys. Rev. E 62(6), 7886-7893.

Arrayás, M., Kaufman, I. K., Luchinsky, D. G., McClintock, P. V. E. \& Soskin, S. M. [2000] "The Kramers problem for a multi-well potential", Phys. Rev. Lett. 84(12), $2556-2559$.

Auerbach, D., Cvitanović, P., Eckmann, J.-P., Gunaratne, G. \& Procaccia, I. [1987] "Exploring chaotic motion through periodic orbits", Phys. Rev. Lett. 58(23), 23872389.

Barreto, E., Kostelich, E. J., Grebogi, C., Ott, E. \& Yorke, J. A. [1995] "Efficient switching between controlled unstable periodic orbits in higher dimensional chaotic systems", Phys. Rev. E 51(5), 4169-4172.

Beale, P. D [1989] "Noise-induced escape from attractor in one-dimensional maps", Phys. Rev. A 40(7), 3998-4003.

Boccaletti, S., Grebogy, C., Lai, Y.-C., Mancini, H. \& Maza, D. [2000] "The control of chaos: theory and applications", Phys. Rep. 329, 103-197.

Cash, J. R. [1986] "On the numerical integration of nonlinear two-point boundary value problems using iterated deferred corrections, part 1: A survey and comparison of some one-step formulae", Comput. Math. Appl. A 12(10), 1029-1048.

Cash, J. R. [1988] "On the numerical integration of nonlinear two-point boundary value problems using iterated deferred corrections, part 2: The development and analysis of highly stable deferred correction formulae", SIAM J. Numer. Anal. 25(4), 862-882.

Cash, J. R. \& Wright, M. H. [1990] "Implementation issues in solving nonlinear equations for two-point boundary value problems", Computing 45(1), 17-37.

Cash, J. R. \& Wright, M. H. [1991] "A deferred correction method for nonlinear two-point boundary-value-problems: Implementation and numerical evaluation", SIAM J. Sci. Stat. Comput. 12(4), 971-989.

Chizhevsky, V. N., Grigorieva, E. V. \& Kashchenko, S. [1997] "Optimal timing for targeting periodic orbits in a loss-driven $\mathrm{CO}_{2}$ laser", Opt. Commun. 133(1-6), 189-195.

Chizhevsky, V. N. \& Turovets, S. I. [1994] "Optimal timing for targeting periodic orbits in a loss-driven $\mathrm{CO}_{2}$ laser", Phys. Rev. A 50(2), 1840-1843. 
Corbalán, R., Cortit, J., Pisarchik, A. N., Chizhevsky, V. N. \& Vilaseca, R. [1995] "Investigation of $\mathrm{CO}_{2}$ laser response to loss perturbation near period doubling", Phys. Rev. A 51(1), 663-668.

Dhamala, M. \& Lai, Y. C. [1999] "Unstable periodic orbits and the natural measure of nonhyperbolic chaotic saddles", Phys. Rev. E 60(5), 6176-6179.

Dreyfus, S. E. [1965] "Dynamic programming and the calculus of variations", Math in science and engineering $\mathbf{2 1}$ Academic Press, New York.

Dykman, M. I. \& Krivoglaz, M. A. [1984] "Theory of nonlinear oscillator interacting with a medium", In Khalatnikov, I.M., editor, Soviet Physics Reviews, volume 5, pp 265-442. Harwood Academic Publishers, New York.

Dykman, M. I., McClintock, P. V. E., Smelyanskiy, V. N., Stein, N. D. \& Stocks, N. G. [1992] "Optimal paths and the prehistory problem for large fluctuations in noise driven systems", Phys. Rev. Lett. 68(18), 2718-2721.

Dykman, M. I., Millonas, M. M. \& Smelyanskiy, V. N. [1994] "Observable and hidden features of large fluctuations in nonequilibrium systems", Phys. Lett. A 195(1), 53-58.

Dykman, M. I., Mori, E., Ross, J. \& Hunt, P. M. [1994] "Large fluctuations and optimal paths in chemical kinetics", J Chem. Phys. 100(8), 5735-5750.

Dykman, M. I., Luchinsky, D. G., McClintock, P. V. E. \& Smelyanskiy, V. N. [1996] "Corrals and CriticalBehavior of the Distribution of Fluctuational Paths", Phys. Rev. Lett. $77(27)$, 5229-5232.

Dykman, M. I., Rabitz, H., Smelyanskiy, V. N. \& Vugmeister, B. E. [1997] "Resonant directed diffusion in nonadiabatically driven systems", Phys. Rev. Lett. 79(7), 11781181 .

Einchcomb, S. J. B. \& McKane, A. J. [1995] "Use of Hamiltonian mechanics in systems driven by colored noise", Phys. Rev. E 51(4), 2974-2981.

Feudel, U. \& Grebogi, C. [1997] "Multistability and control of complexity", Chaos 7(4), $597-604$.

Feudel, U., Grebogi, C., Poon, L. \& Yorke, J. A. [1998] "Dynamical properties of a simple mechanical system with a large number of coexisting periodic attractors", Chaos, Solitons \&6 Fractals 9(1/2), 171-180.

Feynman, R. P. \& Hibbs, A. R. [1965] "Quantum Mechanics and Path Integrals. McGrawHill, New-York. 
Fleming, W. H. [1978] "Exit probabilities and stochastic control", Appl. Math. Optim. 4, 329-346.

Freidlin, M. \& Wentzel, A. D. [1984] Random Perturbations in Dynamical Systems. Springer, New-York.

Gadaleta, S., \& Dangelmayr, G. [2001] "Learning to control a complex multistable system", Phys. Rev. E 63036217.

Graham, R., \& Tel, T. [1984] "Existence of a potential for dissipative dynamical systems", Phys. Rev. Lett. 52(24), 9-12.

Graham, R., Hamm, A. \& Tel, T. [1991] "Nonequilibrium potentials for dynamical systems with fractal attractors or repellers", Phys. Rev. Lett. 66(24), 3089-3092.

Grassberger, P. [1989] "Noise-induced escape from attractors", J. Phys. A: Math. Gen. 22(16), 3283-3290.

Grebogi, C., Ott, E. \& Yorke, J. A. [1988] "Unstable periodic orbits and the dimensions of multifractal chaotic attractors", Phys. Rev. A 37(5), 1711-1724.

Hocking, L. M. [1997] Optimal Control. Oxford Appl. Math and Computing Science. Clarendon Press, Oxford.

Hubinger, B., Doerner, R. \& Martienssen, W. [1994] "Controlling chaos experimentally in systems exhibiting large effective Lyapunov exponents", Phys. Rev. E 50(2), 932-948.

Jackson, E. A. [1997] "The OPCL control methods for entrainment, model-resonance, and migration actions on multiple-attractor systems", Chaos 7(4), 550-559.

Jauslin, H. R. [1987] "Nondifferentiable potentials for nonequilibrium steady states", Physica A 144, 179-191.

Kautz, R. L. [1987] "Activation energy for thermally induced escape from a basin of attraction", Phys. Lett. A 125(6,7), 315-319.

Khovanov, I.A., Luchinsky, D.G., Mannella, R. \& McClintock, P.V.E. [2000a] "Fluctuations and the energy-optimal control of chaos", Phys. Rev. Lett. 85(10), 2100-2103.

Khovanov, I.A., Luchinsky, D.G., Mannella, R. \& McClintock, P.V.E. [2000b] "Fluctuational escape from a chaotic attractor", in Stochastic Processes in Physics, Chemistry and Biology, Lecture Notes in Physics, LNP 557, ed. J A Freund and T Pöeschl, Springer-Verlag, Berlin, pp 378-389.

Kifer, Y. [1990a] "A discrete-time version of the Wentzel-Friedlin theory", The Annals of Probab. 18(4), 1676-1692. 
Kifer, Y. [1990b] "Large deviations in dynamical systems and stochastic processes", Trans. Amer. Math. Soc. 321(2), 505-524.

Kraut, S., Feudel, U. \& Grebogi, C. [1999] "Preference of attractors in noisy multistable systems", Phys. Rev. E 59(5), 5253-5260.

Landau, L. D. \& Lifshitz, E. M. [1980] Statistical Physics. Pergamon, New York, 3rd edition. Part 1.

Luchinsky, D. G. [1997] "On the nature of large fluctuations in equilibrium systems: observation of an optimal force", J. Phys. A 30(16), L577-L583.

Luchinsky, D. G., Maier, R. S., Mannella, R., McClintock, P. V. E. \& Stein, D. L. [1999] "Observation of saddle-point avoidance in noise-induced escape", Phys. Rev. Lett. 82(9), 1806-1809.

Luchinsky, D. G., Khovanov, I. A. [1999] "Fluctuation-induced escape from the basin of attraction of a quasiattractor", JETP Lett. 69(11), 825-830.

Luchinsky, D. G. \& McClintock, P. V. E. [1997] "Irreversibility of classical fluctuations studied in analogue electrical circuits", Nature 389(6650), 463-466.

Luchinsky, D. G., McClintock, P. V. E. \& Dykman, M. I. [1998] "Analogue studies of nonlinear systems", Reports on Progress in Physics 61(8), 889-997.

Luchinsky, D. G., McClintock, P. V. E., Soskin, S. M. \& Mannella, R. [1996] "Zerodispersion nonlinear resonance in dissipative systems", Phys. Rev. Lett. 76(24), 44534457.

Maier, R. S. and Stein, D. L. [1993] "Escape problem for irreversible systems", Phys. Rev. E 48(2), 931-938.

Maier, R. S. and Stein, D. L. [1996] "A scaling theory of bifurcations in the symmetric weak-noise escape problem", J Stat. Phys. 83(3-4), 291-357.

Maier, R. S. and Stein, D. L. [1997] "Limiting exit location distributions in the stochastic exit problem", SIAM J Appl. Math. 57(3), 752-790 .

Mannella, R. [1997] “" Supercomputation in Nonlinear and Disordered Systems, pages 100-130. World Scientific, Singapore.

Marsaglia, G. \& Tsang, W.-W. [1984] "A fast, easily implemented method for sampling from decreasing or symmetric unimodal density-functions", SIAM J. Sci. Stat. Comput. $5(2), 349$. 
Onsager, L. \& Machlup, S. [1953] "Fluctuations and irreversible processes", Phys. Rev. 91(6), 1505-1512.

Raj, S. P. \& Rajasekar, S. [1997] "Migration control in two coupled Duffing oscillators", Phys. Rev. E 55(5), 6237-6240.

Schmelcher, P. \& Diakonos, F. K. [1997] "Detecting unstable periodic orbits of chaotic dynamical systems", Phys. Rev. Lett. 78(25), 4733-4736.

Shinbrot, T. [1995] "Progress in the control of chaos", Adv. Phys. 44(2), 73-111.

Shinbrot, T., Grebogy, C., Ott, E. \& Yorke, J. [1993] "Using small perturbations to control chaos", Nature (London) 363(6428), 411-471.

Shinbrot, T., Ott, E., Grebogi, C. \& Yorke, A. J. [1990] "Using chaos to direct trajectories to targets", Phys. Rev. Lett. 65(26), 3215-3218.

Simonovic, N. S. [1999] "Calculations of periodic orbits: The monodromy method and application to regularized systems", Chaos 9(4), 854-864.

Smelyanskiy, V. N. \& Dykman, M. I. [1997] "Optimal control of large fluctuations", Phys. Rev. E 55(3), 2516-2521.

Smelyanskiy, V. N., Dykman, M. I. \& Maier, R. S. [1997] "Topological features of large fluctuations to the interior of a limit cycle", Phys. Rev. E 55(3), 2369-2391.

Soskin, S. M., Luchinsky, D. G., Mannella, R., Neiman, A. B. \& McClintock, P. V. E. [1997] "Zero-dispersion nonlinear resonance", Int. J. of Bifurc. and Chaos 7(4), 923936.

Whittle, P. [1996] Optimal Control Basics and Beyond. John Wiley, Chichester.

Zwanzig, R. [1973] "Nonlinear generalized Langevin equations", J. Stat. Phys. 9, 215-220. 


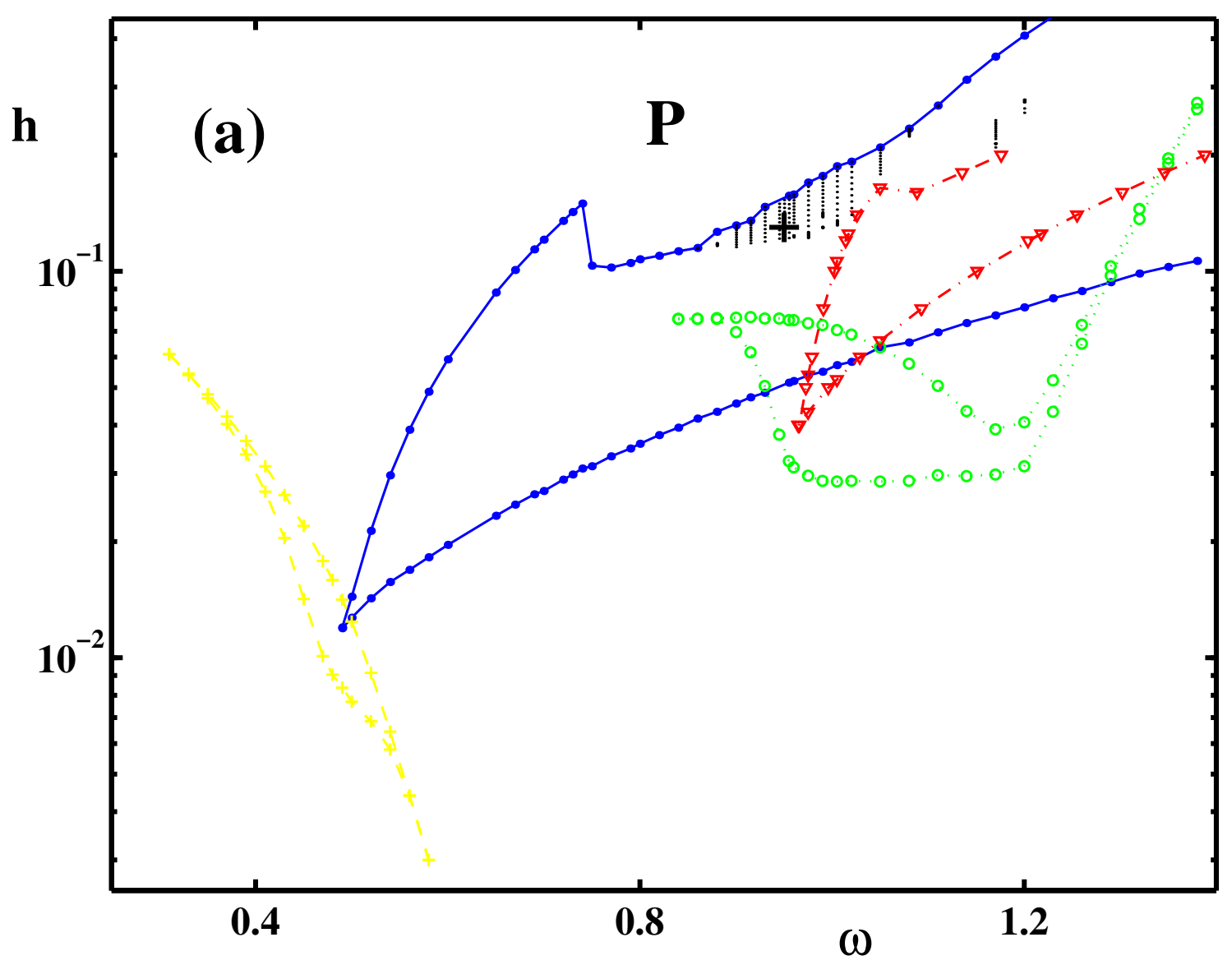

Figure 1: (a) Phase diagram of the system $(5)$ in the $(\omega, h)$ plane obtained in numerical simulations for the parameter values $\Gamma=0.025, \omega_{0}=0.597, \beta=1, \gamma=1$. Regions where chaos was found are shown by the vertical lines of small black dots. The filled blue circles enclose the regions where two nonlinear responses of period 1 coexist. The yellow pluses indicate the boundaries of the hysteretic creation and annihilation of nonlinear responses of period 1 , and the green circles show those for the period 2 resonance. The region where two period 2 attractors coexist is shown by the red triangles. The associated full, dashed-dotted and dashed lines are guides to the eye. The working point $\mathrm{P}$ with $\omega_{f}=0.95, h=0.13$, shown by the large plus, was chosen to lie in the region of coexistence of the period 1 stable limit cycle and of the strange attractor. 


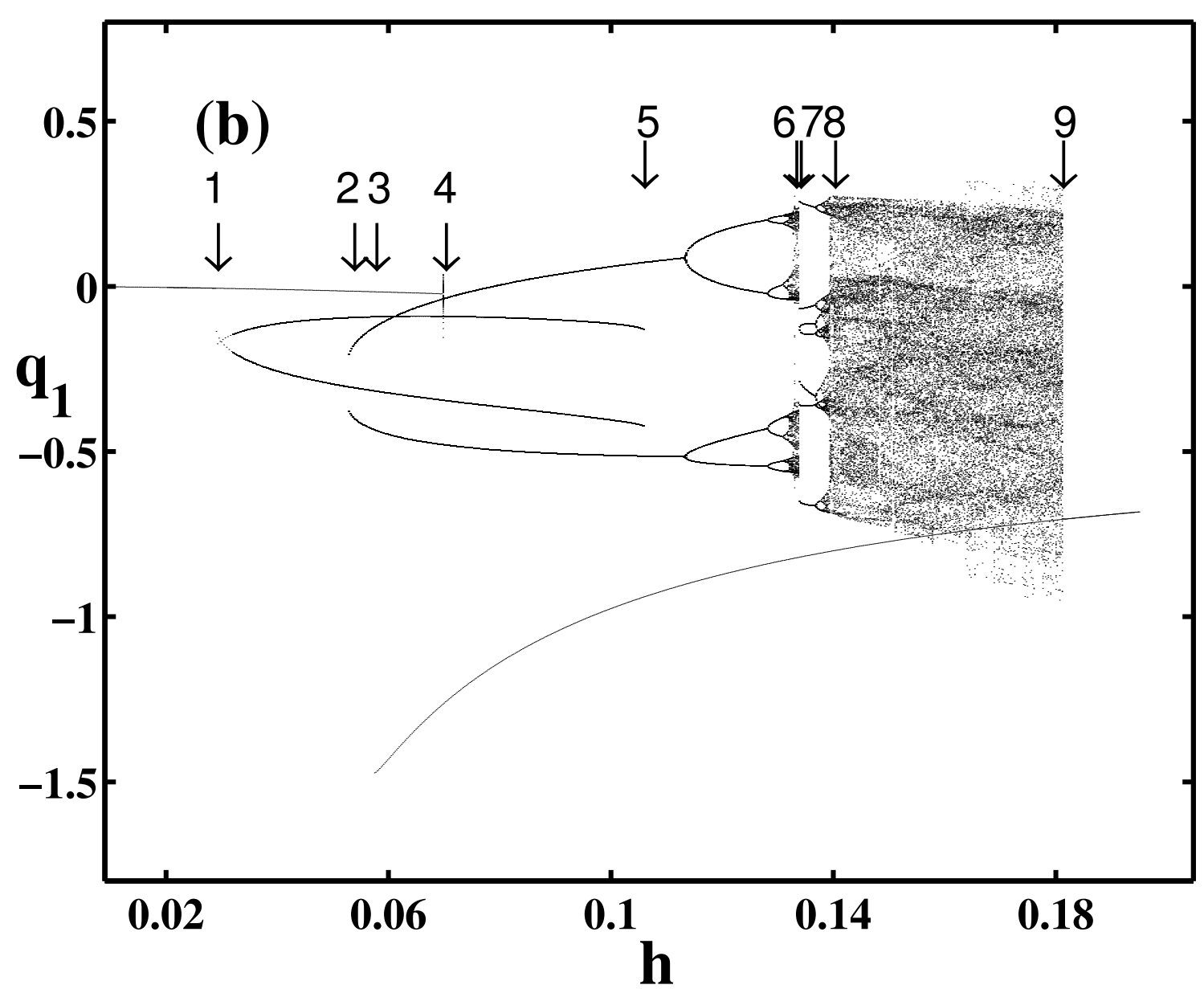

Figure 1: (b) A bifurcation diagram for (5) in Poincaré cross-section obtained with $\omega_{f} t=$ $0(\bmod 2 \pi), \omega_{f}=1.005$ shows values of $q_{1}$ for different $h$. The region of hysteresis for the period 2 resonance lies between the arrows 1 and 4 . The region of coexistence of the two resonances of period 2 lies between arrows 2 and 5; that of the large stable limit cycle of period 1 lies between arrows 3 and 9. Arrows 6-9 show the boundaries of the chaotic states. 


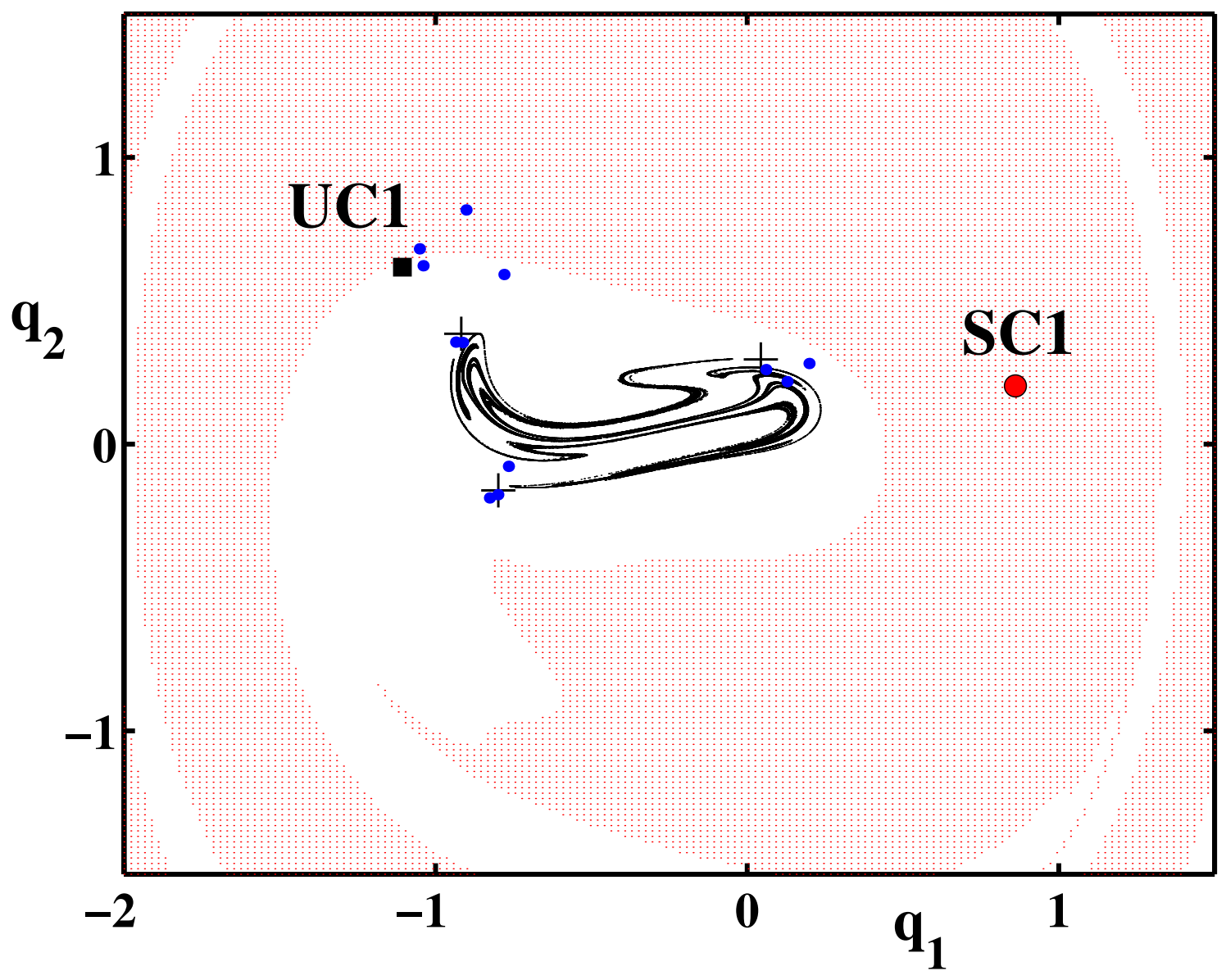

Figure 2: The basin of attraction (shaded red) of the stable limit cycle SC1 (filled red circle) and that of the chaotic attractor CA (white) in Poincaré cross-section with $\omega_{f} t=$ $0.6 \pi(\bmod 2 \pi), \omega_{f}=0.95$. The boundary $\mathrm{S} 1$ of the CA's basin of attraction, the saddle cycle of period 1 , is shown by the black square. The saddle cycle of period 3 is shown by black pluses. The intersections of the actual escape trajectory with the Poincaré crosssection are indicated by the small blue filled circles. 


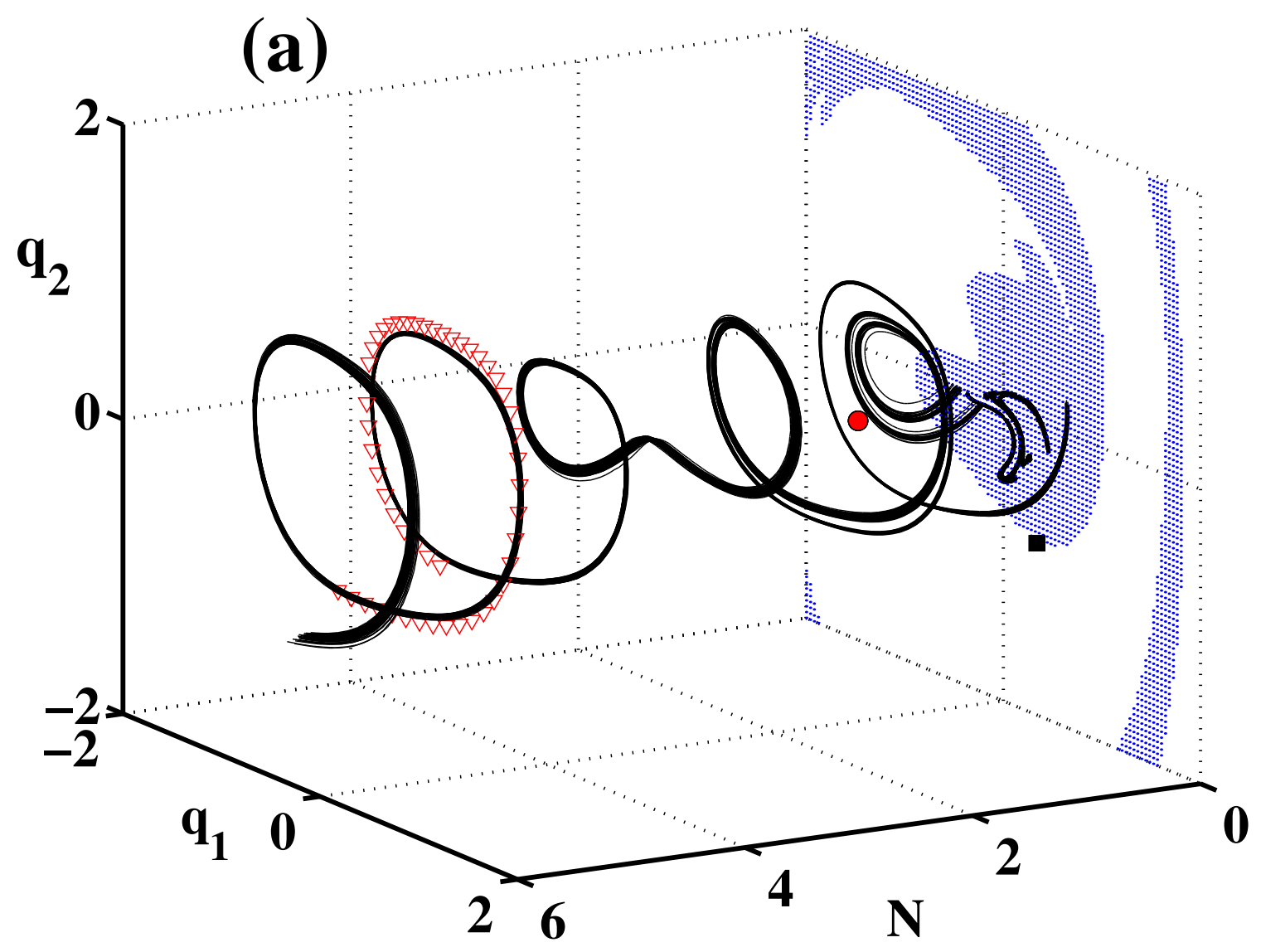

Figure 3: (a) Escape trajectories (black lines) found in the analogue simulations for the parameters $h=0.19, \omega_{f} \approx 1.045, \omega_{0} \approx 0.597, T \approx 0.005$. The red triangles show the calculated saddle cycle of period 1 at the boundary of the basin of attraction. The backplane shows for comparison the Poincaré cross-section and the basins of attraction chaotic attractor (blue-shaded) and stable limit cycle (white) for $\omega_{f} t=0$. The red filled circle and the black filled square indicate respectively the intersections of the stable limit cycle and saddle cycle with the Poincaré section. 


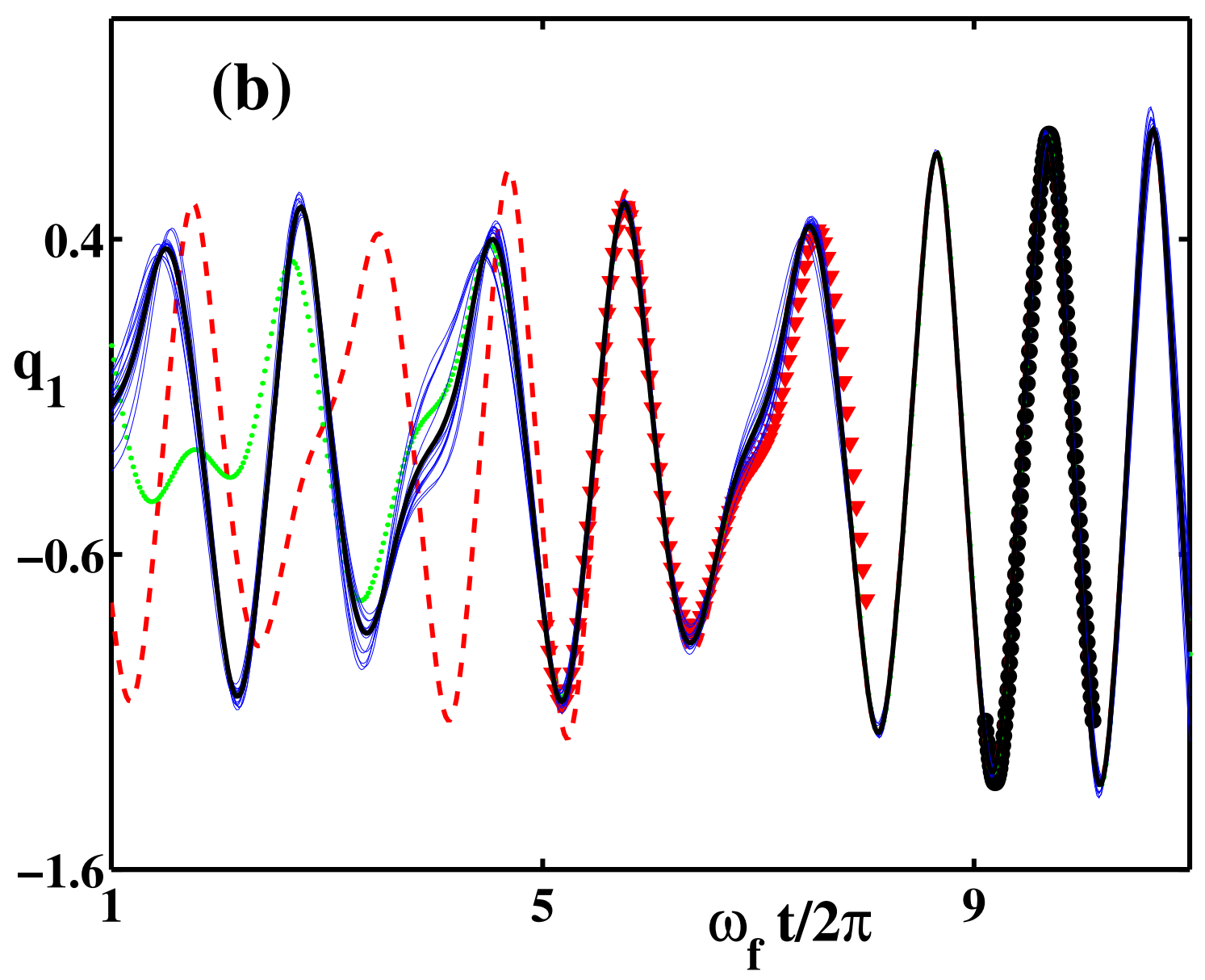

Figure 3: (b) Three optimal escape trajectories obtained in digital simulations for parameters corresponding to the point $\mathrm{P}$ in Fig. 1 with $T \approx 0.005$ are shown by the full black, red dashed solid, and green dotted lines respectively. Their relative probabilities are 9.5:4:1, respectively. The filled circles and triangles show one period of an unstable saddle cycle of period 3 (S3) and one period of an unstable limit cycle S1, respectively. The thin blue lines show 15 real escape trajectories corresponding to the same optimal trajectory. 


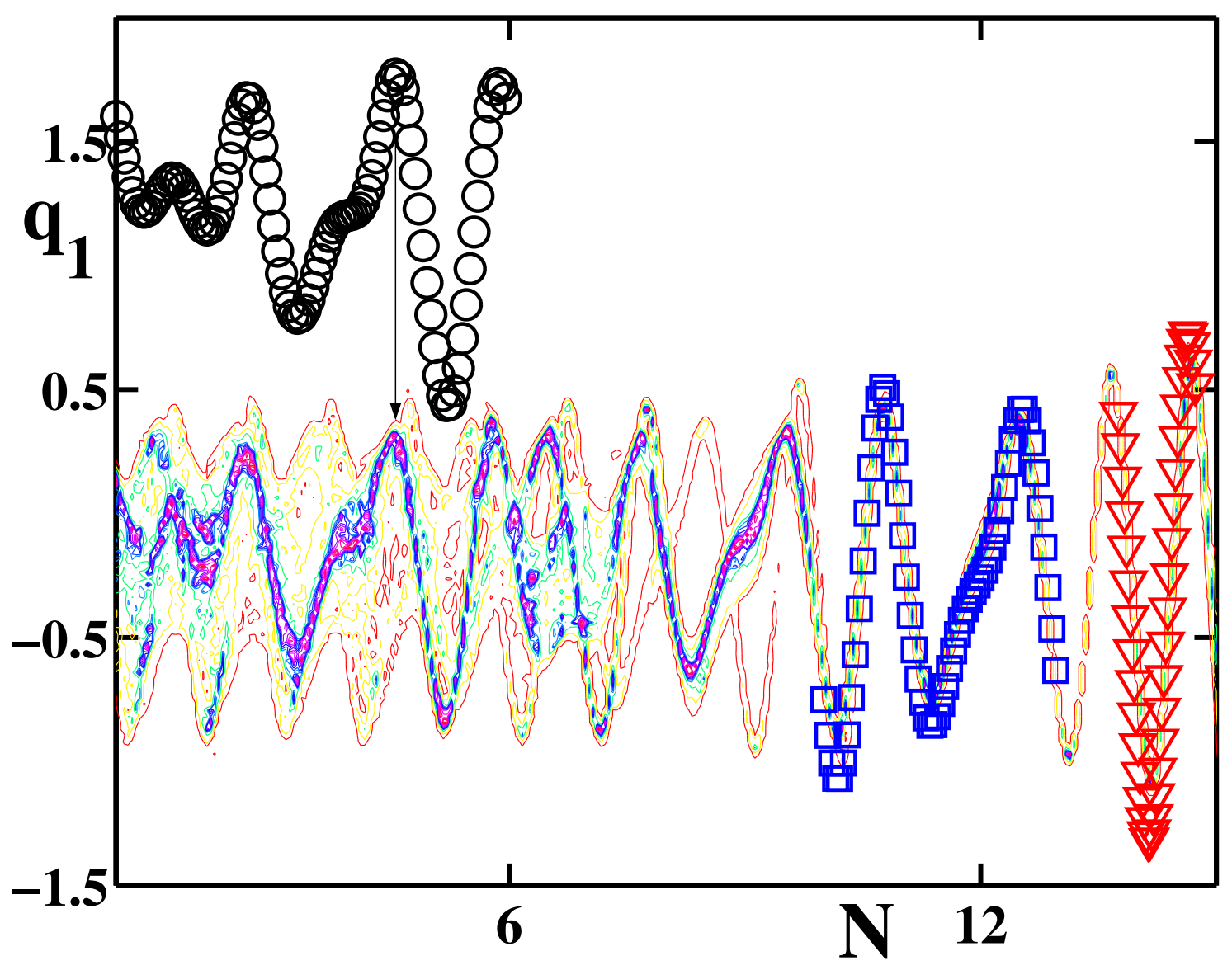

Figure 4: Contour plot of the prehistory probability distribution of escape trajectories for the same parameters as in Fig. 3. The blue squares show one period of the saddle cycle S3. The black circles, showing one period of S5, have been displaced vertically in the interests of clarity. The red triangles indicate the stable limit cycle. 


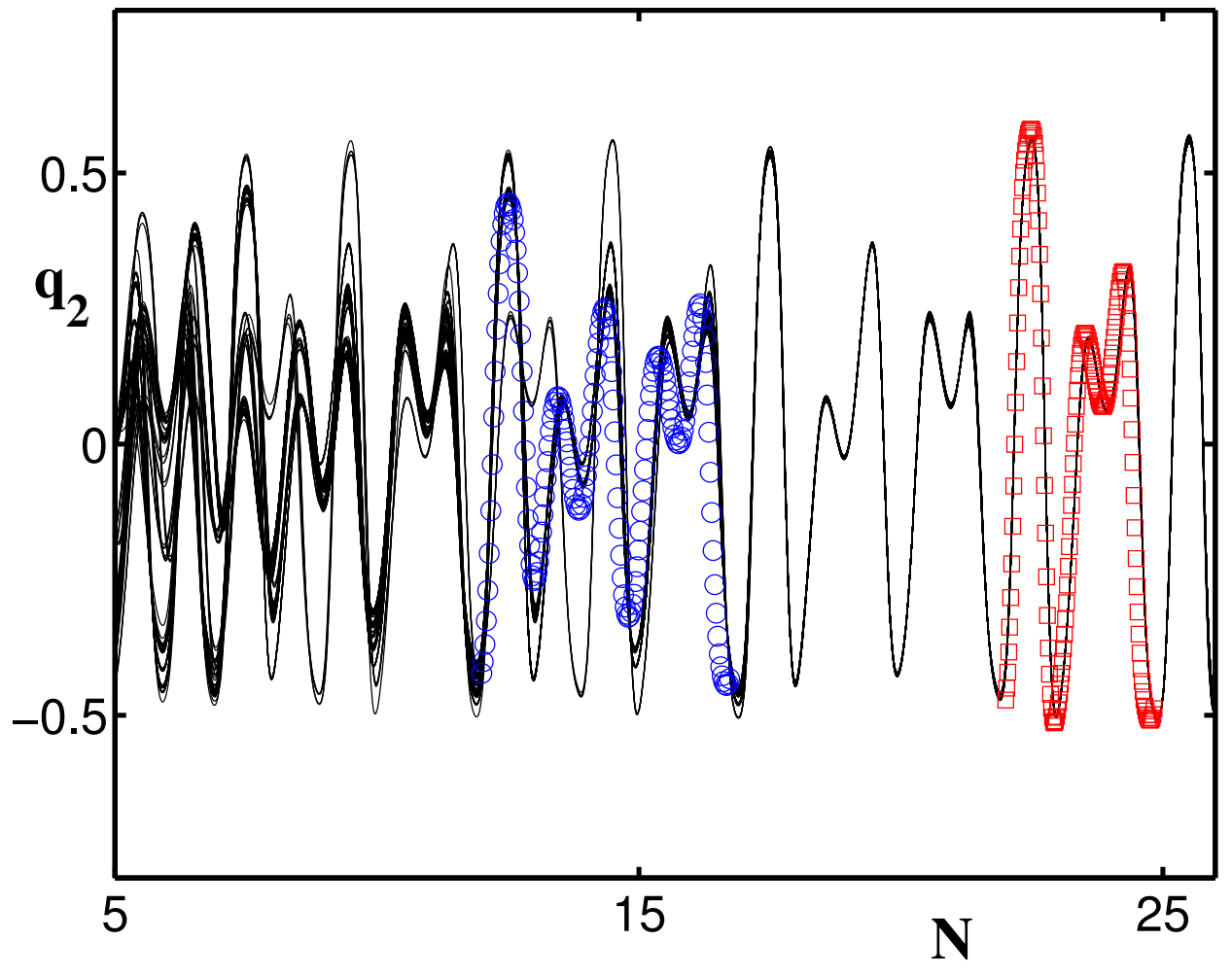

Figure 5: Trajectories merging into the narrow tube around the saddle cycle S3 for the parameters as in Fig. 3a and a temperature of $T=1.5 \times 10^{-6}$. The red squares and blue circles show respectively one period of the saddle cycle S3 and one period of S5. 


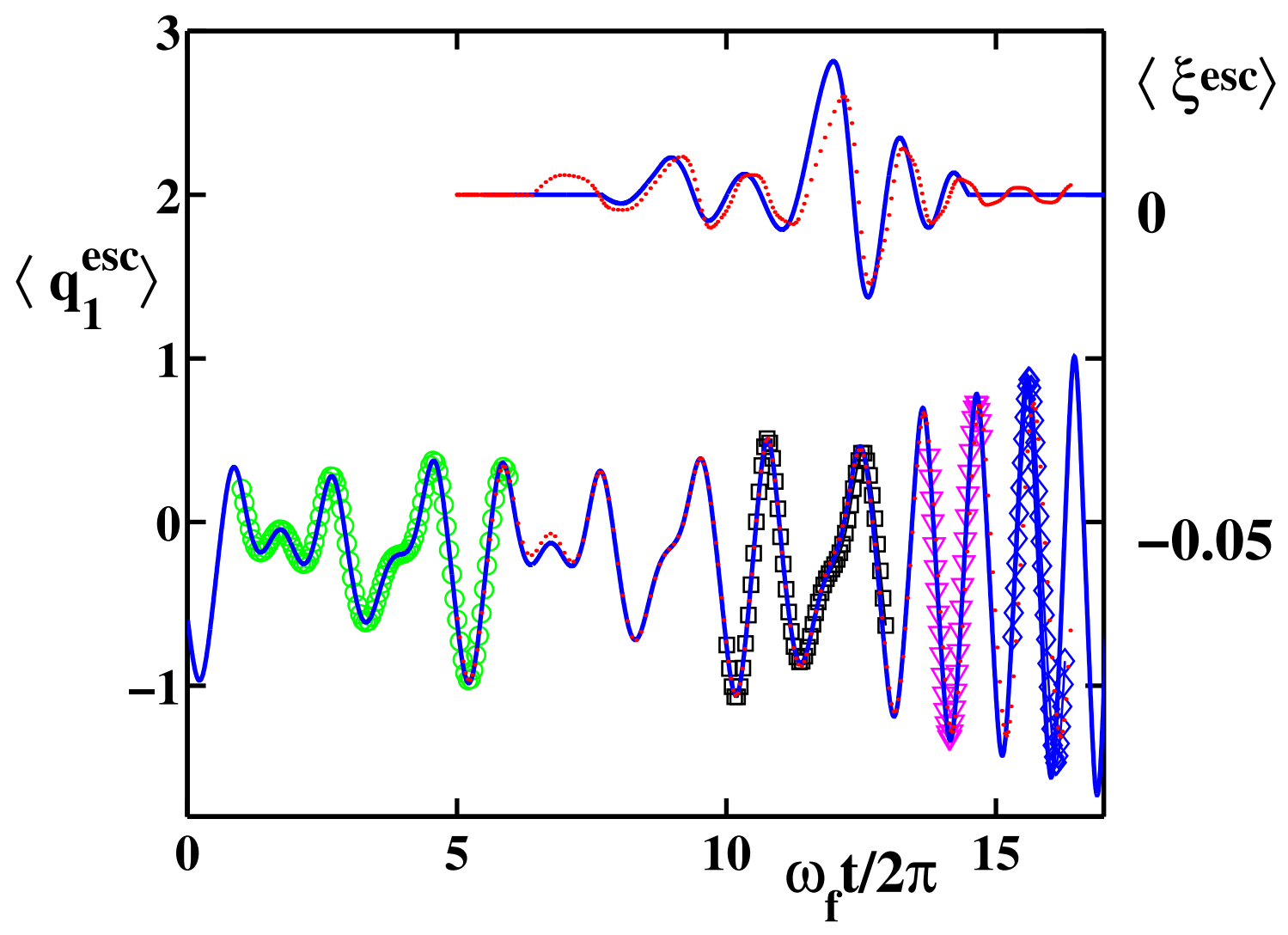

Figure 6: Main figure: the most probable escape path (full blue curve) from S5 to S1, found in the numerical simulations. Single periods of the unstable saddle cycles of period 5, 3 and 1 are shown by green circles, black squares and mauve triangles respectively; the stable limit cycle is shown by blue rhombs. The parameters were $h=0.13, \omega_{f} \approx 0.95$, $\omega_{0} \approx 0.597, D \approx 0.01$. Inset: the optimal force (full blue curve) corresponding to the optimal path, after filtration. The red-dotted line in both parts of the figure is from a numerical solution of the boundary value problem. 


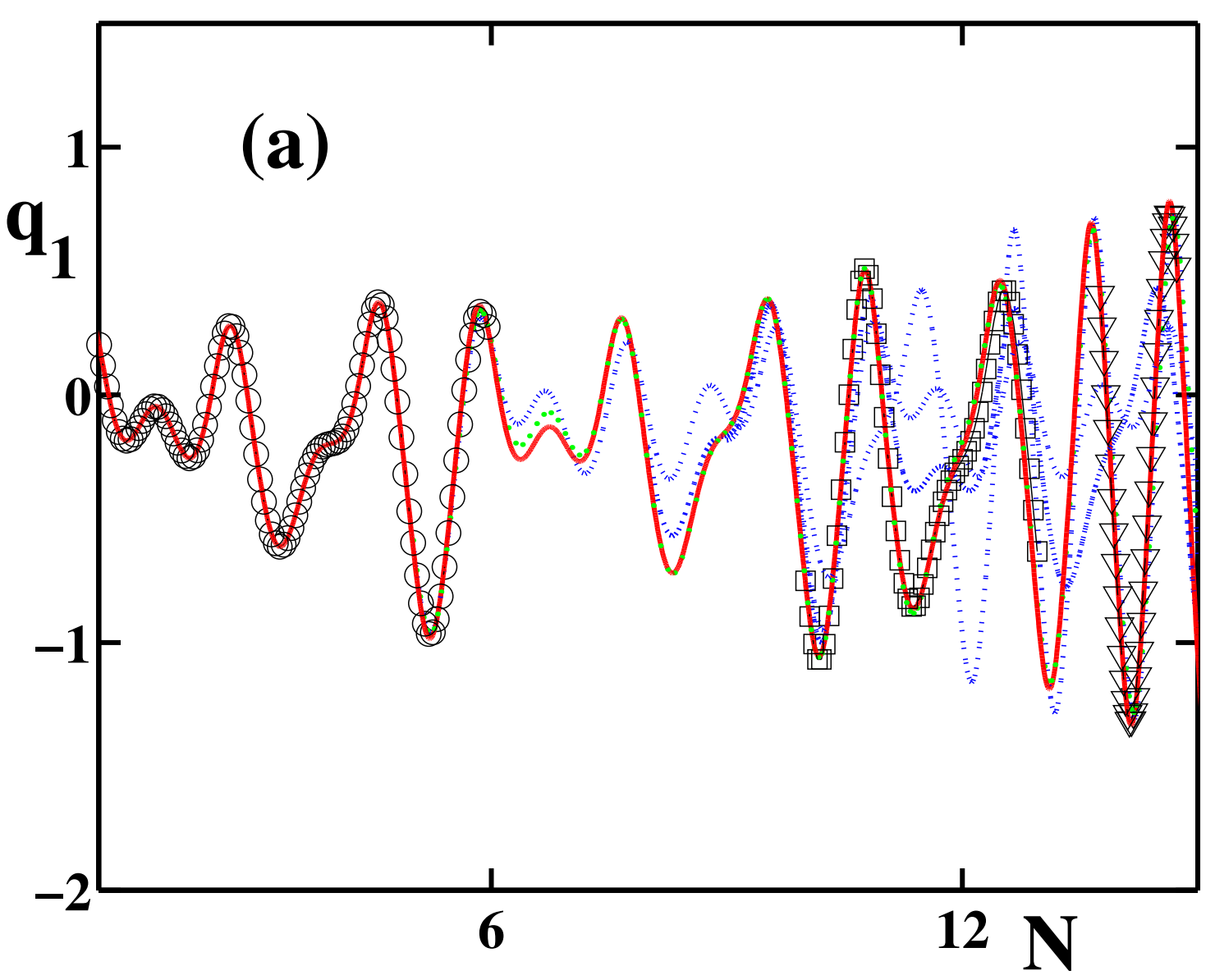

Figure 7: (a) The relaxation of some initial trial functions (blue) onto the optimal path (green dots). The saddle cycles of the period 1, 3 and 5 are shown by the black circles, squares and triangles respectively. The red line represent the optimal fluctuational force found from statistical analysis of the escape trajectories. The parameters were $h=0.13$, $\omega_{f}=0.95, \omega_{0}=0.597$. 


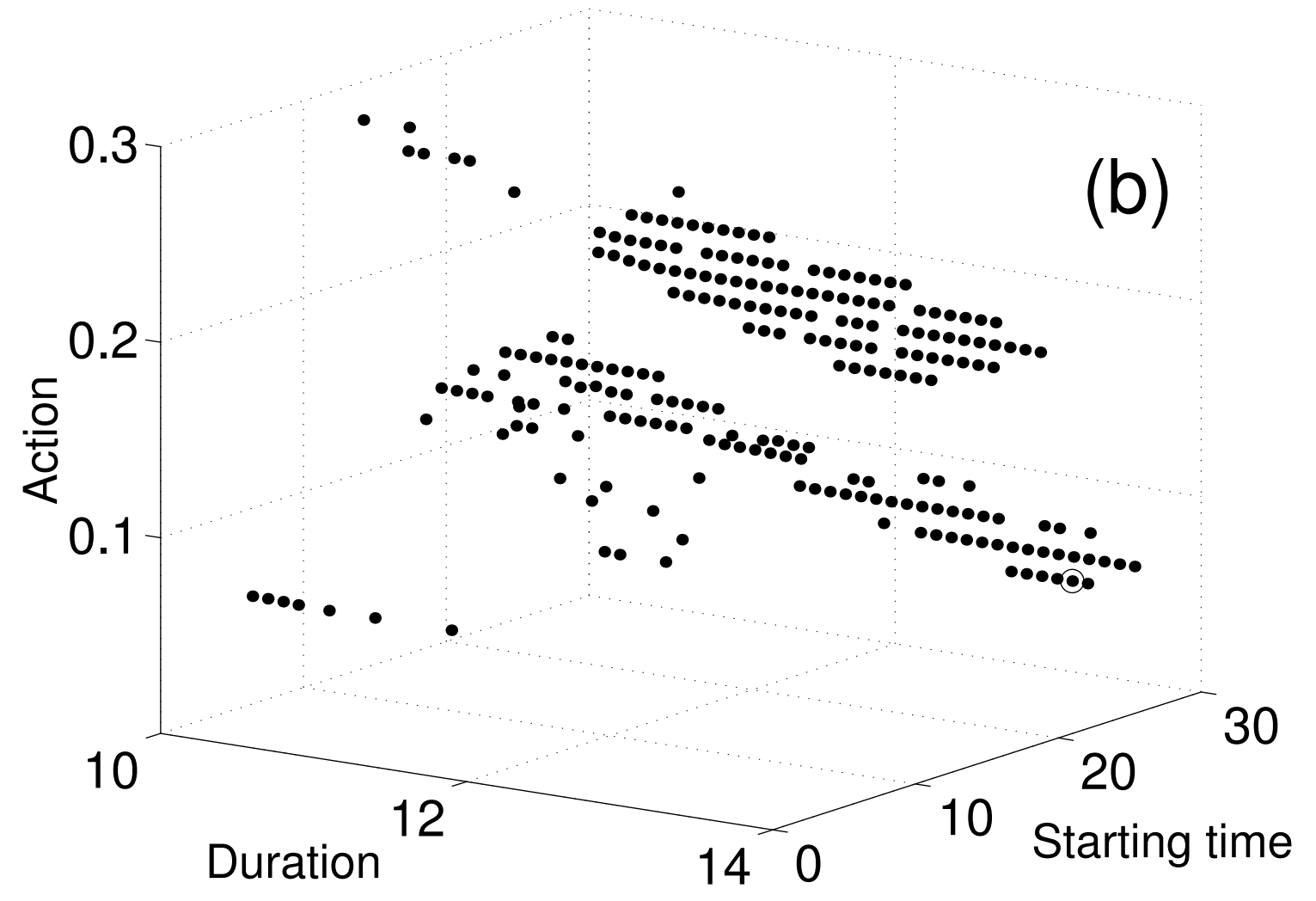

Figure 7: (b) Examples of local minima obtained by the relaxation method as a function of the starting time on the S5 and of the duration of the escape path. 

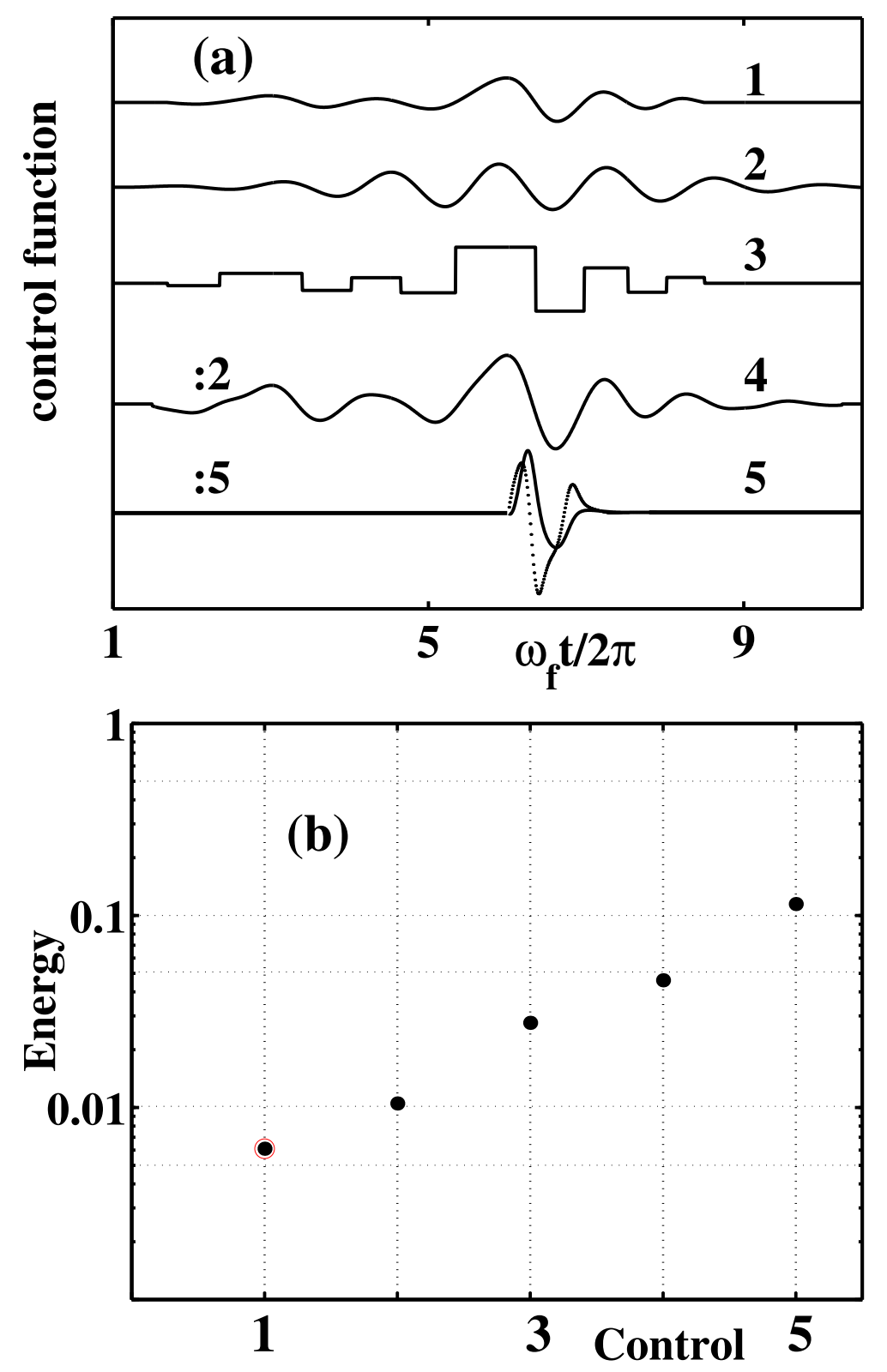

Figure 8: (a) The control functions (not to the same scale) used in the numerical experiments: 1 - optimal force found by statistical analysis of the fluctuational escape trajectories; 2 - approximation of the optimal force by $u(t)=a_{1} \sin \left(a_{2} t\right) \exp \left(-\left(t-a_{3}\right)^{2} a_{4}\right)$ where $a_{i}$ are constants; 3 - approximation of the optimal force by the rectangular pulses; 4 - arbitrary perturbation of the optimal force with a low-frequency perturbation; 5 - control functions produced by the OPCL algorithm. (b) Energies of the control functions shown in (a). 

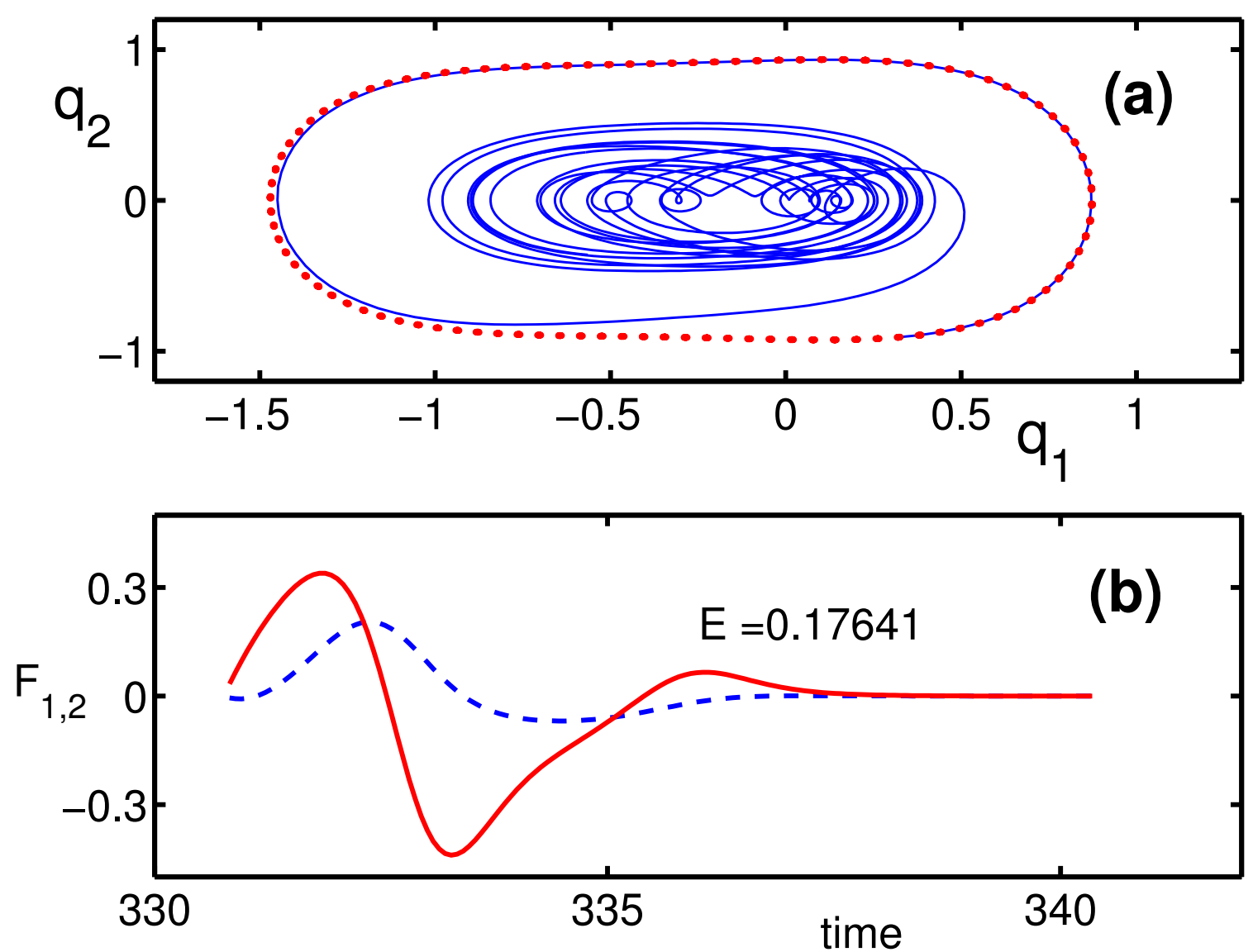

Figure 9: (a) Migration dynamics of the system (21). The blue line is a trajectory taking the system from the chaotic attractor (centre) to the stable limit cycle (red dotted line). The parameters of the OPCL algorithm where: $a_{11}=-2.12, a_{22}=-3.79, \lambda=0.11$. (b) The control functions $F_{1}$ (blue dashed line) and $F_{2}$ (red line). 

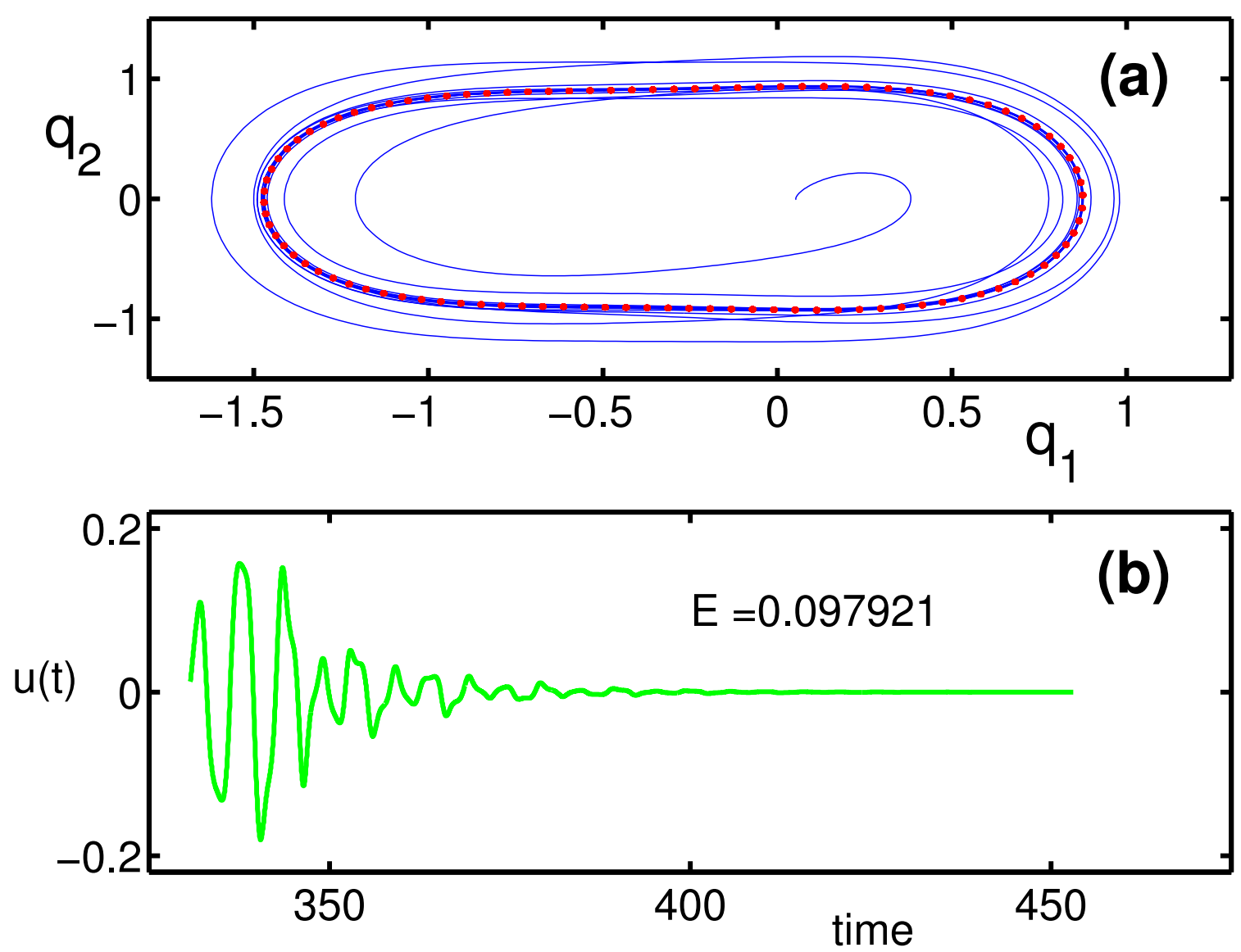

Figure 10: (a) Migration dynamics of the system (5) in the presence of adaptive chaos control. The blue line is a trajectory taking the system from the chaotic attractor (centre) to the stable limit cycle (red dotted line). The parameters of the algorithm where: $\sigma=$ $0.5 \times 10^{-3}, K_{0}=0.15$. (b) The corresponding control function $u(t)$. 